\title{
Geodesics and the competition interface for the corner growth model
}

\author{
Nicos Georgiou ${ }^{1}$ - Firas Rassoul-Agha ${ }^{2}$. \\ Timo Seppäläinen ${ }^{3}$
}

Received: 13 October 2015 / Revised: 10 May 2016 / Published online: 10 August 2016

(C) Springer-Verlag Berlin Heidelberg 2016

\begin{abstract}
We study the directed last-passage percolation model on the planar integer lattice with nearest-neighbor steps and general i.i.d. weights on the vertices, outside the class of exactly solvable models. In Georgiou et al. (Probab Theory Relat Fields, 2016, doi:10.1007/s00440-016-0729-x) we constructed stationary cocycles and Busemann functions for this model. Using these objects, we prove new results on the competition interface, on existence, uniqueness, and coalescence of directional semi-infinite geodesics, and on nonexistence of doubly infinite geodesics.
\end{abstract}

Keywords Busemann function - Coalescence - Cocycle - Competition interface . Directed percolation $\cdot$ Geodesic $\cdot$ Last-passage percolation

Mathematics Subject Classification 60K35 - 65K37

Timo Seppäläinen

seppalai@math.wisc.edu

Nicos Georgiou

n.georgiou@sussex.ac.uk

Firas Rassoul-Agha

firas@math.utah.edu

1 Department of Mathematics, School of Mathematical and Physical Sciences,

University of Sussex, Falmer Campus, Brighton BN1 9QH, UK

2 Mathematics Department, University of Utah, 155 South 1400 East, Salt Lake City, UT 84109, USA

3 Mathematics Department, University of Wisconsin-Madison, Van Vleck Hall, 480 Lincoln Dr., Madison, WI 53706, USA 


\section{Introduction}

In the corner growth model, or directed nearest-neighbor last-passage percolation (LPP) on the lattice $\mathbb{Z}^{2}$, i.i.d. random weights $\left\{\omega_{x}\right\}_{x \in \mathbb{Z}^{2}}$ are used to define last-passage times $G_{x, y}$ between lattice points $x \leq y$ in $\mathbb{Z}^{2}$ by

$$
G_{x, y}=\max _{x} \sum_{k=0}^{n-1} \omega_{x_{k}} .
$$

The maximum is over paths $x_{0}=\left\{x=x_{0}, x_{1}, \ldots, x_{n}=y\right\}$ that satisfy $x_{k+1}-x_{k} \in$ $\left\{e_{1}, e_{2}\right\}$ (up-right paths). Geodesics are paths that maximize in (1.1). Geodesics are unique if $\omega_{x}$ has a continuous distribution. For $x \in \mathbb{Z}_{+}^{2}$, the geodesic from 0 to $x$ must go through either $e_{1}$ or $e_{2}$. These two clusters are separated by the competition interface. The purpose of this paper is to study the geodesics and competition interface for the case where the weights are general, subject to a lower bound $\omega_{0} \geq c$ and a moment condition $\mathbb{E}\left|\omega_{0}\right|^{2+\varepsilon}<\infty$. We address the key questions of existence, uniqueness, and coalescence of directional semi-infinite geodesics, nonexistence of doubly infinite geodesics, and the asymptotic direction of the competition interface.

Systematic study of geodesics in percolation began with the work of Licea and Newman [31]. Their seminal work on undirected first-passage percolation, summarized in Newman's ICM paper [36], utilized a global curvature assumption on the limit shape to derive properties of geodesics, and as a consequence the existence of Busemann functions, which are limits of gradients of passage times. Assuming $\omega_{0}$ has a continuous distribution, they proved the existence of a deterministic, full-Lebesguemeasure set of directions for which there is a unique geodesic out of every lattice point and that geodesics in a given direction from this set coalesce. Furthermore, for any two such directions $\eta$ and $\zeta$ there are no doubly infinite geodesics whose two ends have directions $\eta$ and $-\zeta$.

The global curvature assumption cannot as yet be checked in percolation models with general weights, but it can be verified in several models with special features. One such case is Euclidean first passage percolation based on a homogeneous Poisson point process. For this model, Howard and Newman [28] showed that every geodesic has a direction and that in every fixed direction there is at least one geodesic out of every lattice point.

A number of investigators have built on the approach opened up by Newman et al. This has led to impressive progress in understanding geodesics, Busemann functions, coalescence, competition, and stationary processes in directed last-passage percolation models with enough explicit features to enable verification of the curvature assumptions. This work is on models built on Poisson point processes $[8,10,11,37,46]$ and on the corner growth model with exponential weights $[11,12,19,20,38]$. In the case of the exponential corner growth model, another set of tools comes from its connection with an exactly solvable interacting particle system, namely the totally asymmetric simple exclusion process (TASEP).

The competition interface of the exponential corner growth model maps to a secondclass particle in TASEP, so this object has been studied from both perspectives. An early 
result of Ferrari and Kipnis [18] proved that the scaled location of the second-class particle in a rarefaction fan converges in distribution to a uniform random variable. Mountford and Guiol [35] improved this to almost sure convergence with the help of concentration inequalities and the TASEP variational formula from [43]. Ferrari and Pimentel [20] gave a different proof of almost sure convergence by applying the techniques of directed geodesics and then obtained the distribution of the asymptotic direction of the competition interface from the TASEP results of [18].

Subsequently these results on the almost sure convergence of the competition interface and its limiting random angle were extended from the quadrant to larger classes of initial profiles in two rounds: first by [19] still with TASEP and geodesic techniques, and then by [12] by applying their earlier results on Busemann functions [11]. Coupier [14] also relied on the TASEP connection to sharpen the geodesics results of [20]. He showed that there are no triple geodesics (out of the origin) in any direction and that every fixed direction has a unique geodesic.

To summarize, the common thread of the work above is the use of explicit curvature of the limit shape to control directional geodesics. Coalescence of geodesics leads to Busemann functions and stationary versions of the percolation process. In exactly solvable cases, such as the exponential corner growth model, information about the distribution of the Busemann functions is powerful. For example, it enables calculation of the distribution of the asymptotic direction of the competition interface $[12,19,20]$ and to get bounds on the coalescence time of geodesics $[38,46]$.

An independent line of work is that of Hoffman [26,27] on undirected first passage percolation, with general weights and without regularity assumptions on the limit shape. Hoffman [26] proved that there are at least two semi-infinite geodesics by deriving a contradiction from the assumption that all semi-infinite geodesics coalesce. The technical proof involved the construction of a Busemann function. (Garet and Marchand [21] gave an independent proof with a different method.) Hoffman [27] extended this to at least four geodesics. No further information about geodesics was obtained. In another direction, [44] restricted the number of doubly infinite geodesics to zero or infinity.

The idea of studying geodesic-like objects to produce stationary processes has also appeared in random dynamical systems. Article [17] and its extensions [5, 6,9,25,29] prove existence and uniqueness of semi-infinite minimizers of an action functional to conclude existence and uniqueness of an invariant measure for the Burgers equation with random forcing. These articles treat situations where the space is compact or essentially compact. To make progress in the non-compact case, the approach of Newman et al. was adopted again in $[7,8]$, as mentioned above.

A new approach to the problem of geodesics came in the work of Damron and Hanson [15] who constructed (generalized) Busemann functions from weak subsequential limits of first-passage time differences. This gave access to properties of geodesics, while weakening the need for the global curvature assumption. For instance, assuming differentiability and strict convexity of the limit shape, [15] proves that, with probability one, every semi-infinite geodesic has a direction and for any given direction there exists a semi-infinite directed geodesic out of every lattice point. They construct a tree of semi-infinite geodesics in any given direction such that from every lattice point emanates a unique geodesic in this tree and the tree has no doubly infinite geo- 
desics. However, since the Busemann functions of [15] are constructed from weak subsequential limits, no claims about uniqueness of directional geodesics are made. The geodesics constructed in their trees all coalesce, but one cannot infer from this that all geodesics in a given direction coalesce.

When first-passage percolation is restricted to the upper half plane, [45] was the first to rule out the existence of doubly infinite geodesics. Auffinger et al. [4] extended this half-plane result to more general weight distributions and then applied it to prove coalescence in a tree of geodesics constructed through a limit, as in [15] discussed above. The constructed tree of geodesics again has no infinite backward paths, but it is open to show that the geodesics are asymptotically directed in direction $e_{1}$.

The approach of our work is the opposite of the approach that relies on global curvature, and closer in spirit to [15]. We begin by constructing the stationary versions of the percolation process in the form of stationary cocycles. This comes from related results in queueing theory $[32,39]$. Local regularity assumptions on the limit shape then give enough control to prove that these cocycles are also almost sure Busemann functions. This was done in [23].

In the present paper we continue the project by utilizing the cocycles and the Busemann functions to study geodesics and the competition interface of the corner growth model with general weights. In other words, what is achieved here is a generalization of the results of $[14,20]$ without the explicit solvability framework.

A key technical point is that a family of cocycle geodesics can be defined locally by following minimal gradients of a cocycle. The coalescence proof of [31] applies to cocycle geodesics. Monotonicity and continuity properties of these cocycle geodesics allow us to use them to control all geodesics. In the end we reproduce many of the basic properties of geodesics, some with no assumptions at all and others with local regularity assumptions on the limit shape. Note that, in contrast with the results for the explicitly solvable exponential case, our results must take into consideration the possibility of corners and linear segments in the limit shape.

To control the competition interface we characterize it in terms of the cocycles, as was done in terms of Busemann functions in [12,20,37]. Here again we can get interesting results even without regularity assumptions. For example, assuming that the weight $\omega_{x}$ has continuous distribution, the atoms of the asymptotic direction of the competition interface are exactly the corners of the limit shape. Since the shape is expected to be differentiable, the conjecture is that the asymptotic direction has continuous distribution.

To extend our results to ergodic weights and higher dimensions, a possible strategy that avoids the reliance on queueing theory would be to develop sufficient control on the gradients $G_{x,\lfloor n \xi\rfloor}-G_{y,\lfloor n \xi\rfloor}$ (or their point-to-line counterparts) to construct cocycles through weak limits as $n \rightarrow \infty$. This worked well for undirected first-passage percolation in [15] because the gradients are uniformly integrable. Note however that when $\left\{\omega_{x}\right\}$ are only ergodic, the limiting shape can have corners and linear segments, and can even be a finite polygon.

Organization of the paper. Section 2 describes the corner growth model and the main results of the paper. Section 3 states the existence and properties of the cocycles and Busemann functions on which all the results of the paper are based. Section 4 studies 
cocycle geodesics and proves our results for geodesics. Section 5 proves results for the competition interface. Section 6 derives the distributions of the asymptotic speed of the left and right competition interfaces for the corner growth model with geometric weights. This is an exactly solvable case, but this particular feature has not been calculated in the past. Appendix A has auxiliary results such as an ergodic theorem for cocycles proved in [24].

Notation and conventions. $\mathbb{R}_{+}=[0, \infty), \mathbb{Z}_{+}=\{0,1,2,3, \ldots\}, \mathbb{N}=\{1,2,3, \ldots\}$. The standard basis vectors of $\mathbb{R}^{2}$ are $e_{1}=(1,0)$ and $e_{2}=(0,1)$ and the $\ell^{1}$-norm of $x \in \mathbb{R}^{2}$ is $|x|_{1}=\left|x \cdot e_{1}\right|+\left|x \cdot e_{2}\right|$. For $u, v \in \mathbb{R}^{2}$ a closed line segment on $\mathbb{R}^{2}$ is denoted by $[u, v]=\{t u+(1-t) v: t \in[0,1]\}$, and an open line segment by ]$u, v[=\{t u+(1-t) v: t \in(0,1)\}$. Coordinatewise ordering $x \leq y$ means that $x \cdot e_{i} \leq y \cdot e_{i}$ for both $i=1$ and 2. Its negation $x \not \leq y$ means that $x \cdot e_{1}>y \cdot e_{1}$ or $x \cdot e_{2}>y \cdot e_{2}$. An admissible or up-right finite path $x_{0, n}=\left(x_{k}\right)_{k=0}^{n}$, infinite path $x_{0, \infty}=\left(x_{k}\right)_{0 \leq k<\infty}$, or doubly infinite path $x_{-\infty, \infty}=\left(x_{k}\right)_{k \in \mathbb{Z}}$ on $\mathbb{Z}^{2}$ satisfies $x_{k}-x_{k-1} \in\left\{e_{1}, e_{2}\right\} \forall k$.

The basic environment space is $\Omega=\mathbb{R}^{\mathbb{Z}^{2}}$ whose elements are denoted by $\omega$. There is also a larger product space $\widehat{\Omega}=\Omega \times \Omega^{\prime}$ whose elements are denoted by $\widehat{\omega}=\left(\omega, \omega^{\prime}\right)$.

A statement that contains \pm or $\mp$ is a combination of two statements: one for the top choice of the sign and another one for the bottom choice.

\section{Main results}

\subsection{Assumptions}

The two-dimensional corner growth model is the last-passage percolation model on the planar square lattice $\mathbb{Z}^{2}$ with admissible steps $\left\{e_{1}, e_{2}\right\} . \Omega=\mathbb{R}^{\mathbb{Z}^{2}}$ is the space of environments or weight configurations $\omega=\left(\omega_{x}\right)_{x \in \mathbb{Z}^{2}}$. The group of spatial translations $\left\{T_{x}\right\}_{x \in \mathbb{Z}^{2}}$ acts on $\Omega$ by $\left(T_{x} \omega\right)_{y}=\omega_{x+y}$ for $x, y \in \mathbb{Z}^{2}$. Let $\mathfrak{S}$ denote the Borel $\sigma$-algebra of $\Omega$. P is a Borel probability measure on $\Omega$ under which the weights $\left\{\omega_{x}\right\}$ are independent, identically distributed (i.i.d.) nondegenerate random variables with a $2+\varepsilon$ moment. Expectation under $\mathbb{P}$ is denoted by $\mathbb{E}$. For a technical reason we also assume $\mathbb{P}\left(\omega_{0} \geq c\right)=1$ for some finite constant $c$. Here is the standing assumption, valid throughout the paper:

$$
\begin{aligned}
& \mathbb{P} \text { is i.i.d., } \mathbb{E}\left[\left|\omega_{0}\right|^{p}\right]<\infty \text { for some } p>2, \sigma^{2}=\operatorname{Var}\left(\omega_{0}\right)>0 \text {, and } \\
& \mathbb{P}\left(\omega_{0} \geq c\right)=1 \text { for some } c>-\infty
\end{aligned}
$$

The symbol $\omega$ is reserved for these $\mathbb{P}$-distributed i.i.d. weights, also later when they are embedded in a larger configuration $\widehat{\omega}=\left(\omega, \omega^{\prime}\right)$.

The only reason for assumption $\mathbb{P}\left(\omega_{0} \geq c\right)=1$ is that Theorem 3.3 below is proved in [23] by applying queueing theory. In that context $\omega_{x}$ is a service time and the results have been proved only for $\omega_{x} \geq 0$. (The extension to $\omega_{x} \geq c$ is immediate.) Once the queueing results are extended to general real-valued i.i.d. weights $\omega_{x}$ subject to the moment assumption in (2.1), everything in this paper is true for these general real-valued weights. 


\subsection{Last-passage percolation}

Given an environment $\omega$ and two points $x, y \in \mathbb{Z}^{2}$ with $x \leq y$ coordinatewise, define the point-to-point last-passage time by

$$
G_{x, y}=\max _{x_{0, n}} \sum_{k=0}^{n-1} \omega_{x_{k}} .
$$

The maximum is over paths $x_{0, n}=\left(x_{k}\right)_{k=0}^{n}$ that start at $x_{0}=x$, end at $x_{n}=y$ with $n=|y-x|_{1}$, and have increments $x_{k+1}-x_{k} \in\left\{e_{1}, e_{2}\right\}$. We call such paths admissible or up-right.

According to the basic shape theorem (Theorem 5.1(i) of [34]) there exists a nonrandom continuous function $g_{\mathrm{pp}}: \mathbb{R}_{+}^{2} \rightarrow \mathbb{R}$ such that

$$
\lim _{n \rightarrow \infty} n^{-1} \max _{x \in \mathbb{Z}_{2}^{+}:|x|_{1}=n}\left|G_{0, x}-g_{\mathrm{pp}}(x)\right|=0 \quad \mathbb{P} \text {-almost surely. }
$$

The shape function $g_{\mathrm{pp}}$ is symmetric, concave, and 1-homogeneous (i.e. $g_{\mathrm{pp}}(c \xi)=$ $c g_{\mathrm{pp}}(\xi)$ for $\xi \in \mathbb{R}_{+}^{2}$ and $\left.c \in \mathbb{R}_{+}\right)$.

\subsection{Gradients and convexity}

Since $g_{\mathrm{pp}}$ is homogeneous, it is completely determined by its values on $\mathscr{U}=\left\{t e_{1}+\right.$ $\left.(1-t) e_{2}: t \in[0,1]\right\}$, the convex hull of $\mathscr{R}=\left\{e_{1}, e_{2}\right\}$. The relative interior ri $\mathscr{U}$ is the open line segment $\left\{t e_{1}+(1-t) e_{2}: t \in(0,1)\right\}$. Let

$$
\mathscr{D}=\left\{\xi \in \text { ri } \mathscr{U}: g_{\text {pp }} \text { is differentiable at } \xi\right\}
$$

be the set of points at which the gradient $\nabla g_{\mathrm{pp}}(\xi)$ exists in the usual sense of differentiability of functions of several variables. By concavity the set (ri $\mathscr{U}$ ) $\backslash \mathscr{D}$ is at most countable.

A point $\xi \in$ ri $\mathscr{U}$ is an exposed point if there exists a vector $v \in \mathbb{R}^{2}$ such that

$$
\forall \zeta \in(\operatorname{ri} \mathscr{U}) \backslash\{\xi\}: g_{\mathrm{pp}}(\zeta)<g_{\mathrm{pp}}(\xi)+v \cdot(\zeta-\xi)
$$

The set of exposed points of differentiability of $g_{\text {pp }}$ is $\mathscr{E}=\{\xi \in \mathscr{D}:(2.3)$ holds $\}$. For $\xi \in \mathscr{E}$ we have $v=\nabla g_{\text {pp }}(\xi)$ uniquely. Condition (2.3) is formulated in terms of $\mathscr{U}$ because as a homogeneous function $g_{\text {pp }}$ cannot have exposed points on $\mathbb{R}_{+}^{2}$.

It is expected but currently unknown that $g_{\text {pp }}$ is differentiable on all of ri $\mathscr{U}$. But left and right gradients exist. A left limit $\xi \rightarrow \zeta$ on $\mathscr{U}$ means that $\xi \cdot e_{1}$ increases to $\zeta \cdot e_{1}$, while in a right limit $\xi \cdot e_{1}$ decreases to $\zeta \cdot e_{1}$.

For $\zeta \in$ ri $\mathscr{U}$ define one-sided gradient vectors by

$$
\begin{aligned}
& \nabla g_{\mathrm{pp}}(\zeta \pm) \cdot e_{1}=\lim _{\varepsilon \searrow 0} \frac{g_{\mathrm{pp}}\left(\zeta \pm \varepsilon e_{1}\right)-g_{\mathrm{pp}}(\zeta)}{ \pm \varepsilon} \\
& \quad \text { and } \nabla g_{\mathrm{pp}}(\zeta \pm) \cdot e_{2}=\lim _{\varepsilon \searrow 0} \frac{g_{\mathrm{pp}}\left(\zeta \mp \varepsilon e_{2}\right)-g_{\mathrm{pp}}(\zeta)}{\mp \varepsilon}
\end{aligned}
$$


Concavity of $g_{\mathrm{pp}}$ ensures the limits exist. $\nabla g_{\mathrm{pp}}(\xi \pm)$ coincide (and equal $\nabla g_{\mathrm{pp}}(\xi)$ ) if and only if $\xi \in \mathscr{D}$. Furthermore, on ri $\mathscr{U}$,

$$
\begin{gathered}
\nabla g_{\mathrm{pp}}(\zeta-)=\lim _{\xi \cdot e_{1} \nearrow \zeta \cdot e_{1}} \nabla g_{\mathrm{pp}}(\xi \pm), \quad \nabla g_{\mathrm{pp}}(\zeta+)=\lim _{\xi \cdot e_{1} \searrow \zeta \cdot e_{1}} \nabla g_{\mathrm{pp}}(\xi \pm) \\
\text { and } g_{\mathrm{pp}}(\zeta)=\nabla g_{\mathrm{pp}}(\zeta \pm) \cdot \zeta
\end{gathered}
$$

For $\xi \in$ ri $\mathscr{U}$ define maximal line segments on which $g_{\mathrm{pp}}$ is linear, $\mathscr{U} \xi$ - for the left gradient at $\xi$ and $\mathscr{U} \xi+$ for the right gradient at $\xi$, by

$$
\mathscr{U} \xi \pm=\left\{\zeta \in \operatorname{ri} \mathscr{U}: g_{\mathrm{pp}}(\zeta)-g_{\mathrm{pp}}(\xi)=\nabla g(\xi \pm) \cdot(\zeta-\xi)\right\}
$$

Either or both segments can degenerate to a point. Let

$$
\mathscr{U}_{\xi}=\mathscr{U}_{\xi-} \cup \mathscr{U}_{\xi+}=[\underline{\xi}, \bar{\xi}] \quad \text { with } \underline{\xi} \cdot e_{1} \leq \bar{\xi} \cdot e_{1} \text {. }
$$

If $\xi \in \mathscr{D}$ then $\mathscr{U}_{\xi+}=\mathscr{U}_{\xi-}=\mathscr{U}_{\xi}$, while if $\xi \notin \mathscr{D}$ then $\mathscr{U}_{\xi+} \cap \mathscr{U}_{\xi-}=\{\xi\}$. If $\xi \in \mathscr{E}$ then $\mathscr{U}_{\xi}=\{\xi\}$. Notations $\underline{\xi}$ and $\bar{\xi}$ can be iterated: $\underline{\xi}=\underline{\eta}$ for $\eta=\underline{\xi}$ and $\overline{\bar{\xi}}=\bar{\zeta}$ for $\zeta=\bar{\xi}$. If $\underline{\xi} \in \mathscr{D}$ then $\underline{\xi}=\underline{\xi}$ and similarly for $\bar{\xi}$. When needed we use the convention $\mathscr{U}_{e_{i}}=\mathscr{U}_{e_{i} \pm}=\left\{e_{i}\right\}, i \stackrel{\bar{\epsilon}}{\underline{\epsilon}}\{1,2\}$.

For $\zeta \cdot e_{1}<\eta \cdot e_{1}$ in ri $\mathscr{U},[\zeta, \eta]$ is a maximal linear segment for $g_{\mathrm{pp}}$ if $\nabla g_{\text {pp }}$ exists and is constant in $] \zeta, \eta$ [ but not on any strictly larger open line segment in ri $\mathscr{U}$. Then $[\zeta, \eta]=\mathscr{U}_{\zeta+}=\mathscr{U}_{\eta-}=\mathscr{U}_{\xi}$ for any $\left.\xi \in\right] \zeta, \eta[$. If furthermore $\zeta, \eta \in \mathscr{D}$ we say that $g_{\mathrm{pp}}$ is differentiable at the endpoints of this maximal linear segment. This hypothesis appears several times. A linear segment of $g_{\text {pp }}$ must lie in the interior ri $\mathscr{U}$. This is a consequence of Martin's shape universality on the boundary of $\mathbb{R}_{+}^{2}[34$, Theorem 2.4] which states that

$$
g_{\mathrm{pp}}(1, s)=\mathbb{E}\left(\omega_{0}\right)+2 \sigma \sqrt{s}+o(\sqrt{s}) \text { as } s \searrow 0
$$

$g_{\mathrm{pp}}$ is strictly concave if there is no nondegenerate line segment on ri $\mathscr{U}$ on which $g_{\mathrm{pp}}$ is linear.

Exposed points can be characterized as follows. All points of (ri $\mathscr{U}) \backslash \mathscr{D}$ are exposed. A point $\xi \in \mathscr{D}$ is exposed if and only if it does not lie in any closed linear segment of $g_{\mathrm{pp}}$.

\subsection{Geodesics}

For $u \leq v$ in $\mathbb{Z}^{2}$ an admissible path $x_{0, n}$ from $x_{0}=u$ to $x_{n}=v$ (with $n=|v-u|_{1}$ ) is a (finite) geodesic from $u$ to $v$ if

$$
G_{u, v}=\sum_{k=0}^{n-1} \omega_{x_{k}} .
$$


An infinite up-right path $x_{0, \infty}=\left(x_{k}\right)_{0 \leq k<\infty}$ is a semi-infinite geodesic emanating from $u \in \mathbb{Z}^{2}$ if $x_{0}=u$ and for all $j>i \geq 0, x_{i, j}$ is a geodesic between $x_{i}$ and $x_{j}$. Two semi-infinite geodesics $x_{0, \infty}$ and $y_{0, \infty}$ coalesce if there exist $m, n \in \mathbb{Z}_{+}$such that $x_{m+i}=y_{n+i} \forall i \in \mathbb{Z}_{+}$.

For $\xi \in \mathscr{U}$, a geodesic $x_{0, \infty}$ is $\xi$-directed or a $\xi$-geodesic if $x_{n} /\left|x_{n}\right|_{1} \rightarrow \xi$ as $n \rightarrow \infty$. A directed geodesic is $\xi$-directed for some $\xi$. Flat segments of $g_{\text {pp }}$ on $\mathscr{U}$ prevent us from asserting that all geodesics are directed. Hence we say more generally for a subset $\mathscr{V} \subset \mathscr{U}$ that a geodesic $x_{0, \infty}$ is $\mathscr{V}$-directed if all the limit points of $x_{n} /\left|x_{n}\right|_{1}$ lie in $\mathscr{V}$. Recall the definition of $\mathscr{U}_{\xi \pm}$ from (2.6) and $\mathscr{U}_{\xi}=\mathscr{U}_{\xi} \cup \cup \mathscr{U}_{\xi-}$.

Theorem 2.1 (i) The following statements hold for $\mathbb{P}$-almost every $\omega$. For every $u \in \mathbb{Z}^{2}$ and $\xi \in \mathscr{U}$ there exists at least one semi-infinite $\mathscr{U} \xi+$-directed geodesic and at least one semi-infinite $\mathscr{U} \xi-$-directed geodesic starting from u. Every semiinfinite geodesic is $\mathscr{U}_{\xi}$-directed for some $\xi \in \mathscr{U}$.

(ii) If $g_{\mathrm{pp}}$ is strictly concave then, $\mathbb{P}$-almost surely, every semi-infinite geodesic is directed.

(iii) Suppose $\mathbb{P}\left\{\omega_{0} \leq r\right\}$ is a continuous function of $r \in \mathbb{R}$. Fix $\xi \in$ ri $\mathscr{U}$ with $\mathscr{U}_{\xi}=[\underline{\xi}, \bar{\xi}]$ satisfying $\underline{\xi}, \xi, \bar{\xi} \in \mathscr{D}$. Then $\mathbb{P}$-almost surely there is a unique $\mathscr{U} \xi$-directed semi-infinite geodesic out of every $u \in \mathbb{Z}^{2}$ and all these geodesics coalesce. For each $u \in \mathbb{Z}^{2}$ there are at most finitely many sites $v \in \mathbb{Z}^{2}$ such that the unique $\mathscr{U}_{\xi}$-directed semi-infinite geodesic out of $v$ goes through $u$.

Under the hypotheses of part (iii) we have $\mathscr{U}_{\xi}=\mathscr{U}_{\xi}$. So there is no contradiction between parts (i) and (iii).

By (2.8) there are infinitely many distinct sets $\mathscr{U} \xi_{ \pm}$. Hence, without any assumptions on the shape $g_{\mathrm{pp}}$, part (i) implies the existence of infinitely many semi-infinite geodesics from each point $u \in \mathbb{Z}^{2}$. The second part of claim (iii) prevents the existence of doubly infinite geodesics $x_{-\infty, \infty}$ such that $x_{0, \infty}$ is $\mathscr{U} \xi$-directed (a.s. in a fixed direction $\xi$ ). This is not true for all weight distributions (see Example 2.5 below).

For exponentially distributed $\omega_{0}$ the results of Theorem 2.1 appeared earlier as follows. Theorem 2.1(i)-(ii) is covered by Proposition 7 of [20]. Uniqueness and coalescence in part (iii) are in Theorem 1(3) of [14], combined with the coalescence proof of [31] which was adapted to exponential LPP in Proposition 8 of [20]. Nonexistence of doubly infinite geodesics is part of Lemma 2 of [38].

When the distribution of $\omega_{0}$ is not continuous, uniqueness of geodesics (Theorem 2.1(iii)) cannot hold. Then we can consider leftmost and rightmost geodesics. The leftmost geodesic $\underline{x}$. (between two given points or in a given direction) satisfies $\underline{x}_{k}$. $e_{1} \leq x_{k} \cdot e_{1}$ for any geodesic $x$. of the same category. The rightmost geodesic satisfies the opposite inequality.

Theorem 2.2 Fix $\xi \in$ ri $\mathscr{U}$. The following hold almost surely.

(i) Assume $\underline{\xi}$ is not the right endpoint of a linear segment of $g_{\mathrm{pp}}$ (equivalently, $\underline{\xi}=\underline{\xi}$ ).

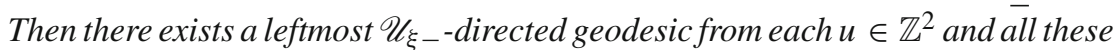
leftmost geodesics coalesce. For each $u \in \mathbb{Z}^{2}$ there are at most finitely many sites

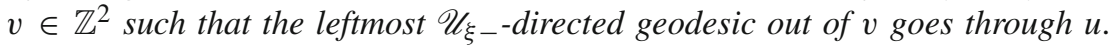
A similar statement holds for rightmost $\mathscr{U} \xi+-$ geodesics, under the assumption $\overline{\bar{\xi}}=\bar{\xi}$. 
(ii) Assume $\xi, \xi, \bar{\xi} \in \mathscr{D}$. Then for any $u \in \mathbb{Z}^{2}$ and sequence $v_{n}$ such that

$$
\left|v_{n}\right|_{1} \rightarrow \infty \text { and } \underline{\xi} \cdot e_{1} \leq \varliminf_{n \rightarrow \infty} \frac{v_{n} \cdot e_{1}}{\left|v_{n}\right|_{1}} \leq \varlimsup_{n \rightarrow \infty} \frac{v_{n} \cdot e_{1}}{\left|v_{n}\right|_{1}} \leq \bar{\xi} \cdot e_{1},
$$

the leftmost geodesic from $u$ to $v_{n}$ converges to the unique leftmost $\mathscr{U} \xi$-directed geodesic from u given in part (i). A similar statement holds for rightmost geodesics.

The convergence statement Theorem 2.2(ii) applies also to the case in Theorem 2.1(iii), and in that case there is just one unique $\mathscr{U}_{\xi}$-directed geodesic, not separate leftmost and rightmost geodesics. Theorems 2.1 and 2.2 are proved in Sect. 4. In particular, we give explicit local recipes in terms of a priori constructed cocycles for defining the geodesics whose existence is claimed in the theorems.

\subsection{Busemann functions and Busemann geodesics}

By (1.1) the following identities hold along any geodesic $x_{0, m}$ from $u$ to $v_{n}$ :

$$
\begin{aligned}
\omega_{x_{i}} & =\min \left(G_{x_{i}, v_{n}}-G_{x_{i}+e_{1}, v_{n}}, G_{x_{i}, v_{n}}-G_{x_{i}+e_{2}, v_{n}}\right) \\
& =G_{x_{i}, v_{n}}-G_{x_{i+1}, v_{n}}, \quad \text { for } \quad 0 \leq i<m .
\end{aligned}
$$

The second equality in (2.10) shows how to construct a finite geodesic ending at $v_{n}$. To study semi-infinite geodesics we take $v_{n} \rightarrow \infty$ in a particular direction. Point-to-point Busemann functions are limits of gradients $G_{x, v_{n}}-G_{y, v_{n}}$. The next existence theorem is Theorem 3.1 from [23].

Theorem 2.3 Fix two points $\zeta, \eta \in \mathscr{D}$ such that $\zeta \cdot e_{1} \leq \eta \cdot e_{1}$. Assume that either

(i) $\zeta=\eta=\xi \in \mathscr{E}$ in which case $\zeta=\eta=\xi=\xi=\overline{\bar{\xi}}$, or that

(ii) $[\zeta, \eta]$ is a maximal linear segment of $g_{\mathrm{pp}}$ in which case $[\zeta, \eta]=[\underline{\xi}, \bar{\xi}]$ for all $\xi \in[\zeta, \eta]$.

Then there exist integrable random variables $\left\{B(x, y): x, y \in \mathbb{Z}^{2}\right\}$ and an event $\Omega_{0}$ with $\mathbb{P}\left(\Omega_{0}\right)=1$ such that the following holds for each $\omega \in \Omega_{0}$. For each sequence $v_{n} \in \mathbb{Z}_{+}^{2}$ such that

$$
\left|v_{n}\right|_{1} \rightarrow \infty \text { and } \zeta \cdot e_{1} \leq \lim _{n \rightarrow \infty} \frac{v_{n} \cdot e_{1}}{\left|v_{n}\right|_{1}} \leq \varlimsup_{n \rightarrow \infty} \frac{v_{n} \cdot e_{1}}{\left|v_{n}\right|_{1}} \leq \eta \cdot e_{1}
$$

we have the limit

$$
B(\omega, x, y)=\lim _{n \rightarrow \infty}\left(G_{x, v_{n}}(\omega)-G_{y, v_{n}}(\omega)\right) \quad \text { for } x, y \in \mathbb{Z}^{2}
$$

The mean of the limit is given by

$$
\nabla g_{\mathrm{pp}}(\xi)=\left(\mathbb{E}\left[B\left(x, x+e_{1}\right)\right], \mathbb{E}\left[B\left(x, x+e_{2}\right)\right]\right) \text { for all } \xi \in[\zeta, \eta]
$$


In particular, suppose $\xi$ is an exposed point of differentiability of $g_{\mathrm{pp}}$, or $\xi$ lies on a maximal linear segment of $g_{\text {pp }}$ whose endpoints are points of differentiability. Then a Busemann function $B^{\xi}$ exists in direction $\xi$ in the sense that $B^{\xi}(\omega, x, y)$ equals the limit in (2.12) for any sequence $v_{n} /\left|v_{n}\right|_{1} \rightarrow \xi$ with $\left|v_{n}\right|_{1} \rightarrow \infty$. Furthermore, the $B^{\xi}$ 's match on maximal linear segments of $g_{\text {pp }}$ with endpoints in $\mathscr{D}$.

Limit (2.12) applied to (2.10) gives

$$
\omega_{x_{i}}=\min _{j \in\{1,2\}} B\left(\omega, x_{i}, x_{i}+e_{j}\right)=B\left(\omega, x_{i}, x_{i+1}\right) \quad \mathbb{P} \text {-a.s. }
$$

The second equality shows how to construct semi-infinite geodesics from a Busemann function. Such geodesics will be called Busemann geodesics. The next theorem shows that in a direction that satisfies the differentiability assumptions that ensure existence of Busemann functions, all geodesics are Busemann geodesics.

Theorem 2.4 Fix $\xi \in$ ri $\mathscr{U}$ with $\mathscr{U} \xi=[\xi, \bar{\xi}]$ such that $\underline{\xi}, \xi, \bar{\xi} \in \mathscr{D}$. Let B be the limit from (2.12). Then there exists an event $\bar{\Omega}_{0}$ with $\mathbb{P}\left(\Omega_{0}\right) \stackrel{-}{=} 1$ and such that statements (i)-(iii) below hold for each $\omega \in \Omega_{0}$.

(i) Every up-right path $x_{0, \infty}$ such that $\omega_{x_{k}}=B\left(x_{k}, x_{k+1}\right)$ for all $k \geq 0$ is a semiinfinite geodesic. We call such a path a Busemann geodesic for $B$.

(ii) Every semi-infinite geodesic $x_{0, \infty}$ that satisfies

$$
\underline{\xi} \cdot e_{1} \leq \lim _{n \rightarrow \infty} \frac{x_{n} \cdot e_{1}}{n} \leq \varlimsup_{n \rightarrow \infty} \frac{x_{n} \cdot e_{1}}{n} \leq \bar{\xi} \cdot e_{1}
$$

is a Busemann geodesic for $B$.

(iii) Let $v_{n}$ be a sequence that satisfies (2.9). Let $m \in \mathbb{N}$. Then $\exists n_{0} \in \mathbb{N}$ such that if $n \geq n_{0}$, then every geodesic $x_{0,\left|v_{n}\right|_{1}}$ from $x_{0}=0$ to $v_{n}$ satisfies $B\left(\omega, x_{i}, x_{i+1}\right)=$ $\omega_{x_{i}}$ for all $i=0,1, \ldots, m$.

Note in particular that the unique geodesics discussed in Theorem 2.1(iii) and Theorem 2.2(ii) are Busemann geodesics. This theorem is proved in Sect. 4.

Example 2.5 (Flat edge in the percolation cone) Assume (2.1) and furthermore that $\omega_{0} \leq 1$ and $\mathbf{p}_{c}<\mathbb{P}\left\{\omega_{0}=1\right\}<1$ where $\mathbf{p}_{c}$ is the critical probability of oriented site percolation on $\mathbb{Z}^{2}$ (see Section 3.2 of [23] for more detail about this setting). Then $g_{\mathrm{pp}}$ has a nondegenerate, symmetric linear segment $[\underline{\eta}, \bar{\eta}]$ such that $\underline{\eta}, \bar{\eta} \in \mathscr{D}[3,16,33]$. According to Theorems 2.2 and 2.4, from any point $u \in \mathbb{Z}^{2}$ there exist unique leftmost and rightmost semi-infinite geodesics directed into the segment $[\eta, \bar{\eta}]$, these geodesics are Busemann geodesics, and finite leftmost and rightmost geodesics converge to these Busemann geodesics.

Note also, in relation to Theorem 2.1(iii), that a doubly infinite geodesic through the origin with $\omega_{x_{k}} \equiv 1$, directed into $[\underline{\eta}, \bar{\eta}]$, can be constructed with positive probability by joining together a percolating path in the first quadrant with one in the third quadrant. 
Fig. 1 The geodesic tree $\mathscr{T}_{0}$ rooted at 0 . The competition interface (solid line) emanates from $\left(\frac{1}{2}, \frac{1}{2}\right)$ and separates the subtrees of $\mathscr{T}_{0}$ rooted at $e_{1}$ and $e_{2}$

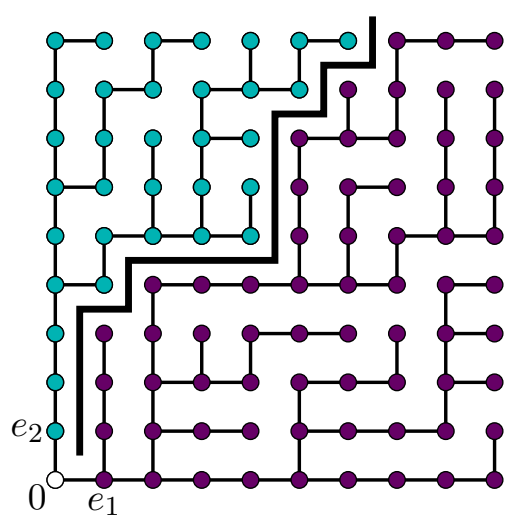

\subsection{Competition interface}

For this subsection assume that $\mathbb{P}\left\{\omega_{0} \leq r\right\}$ is a continuous function of $r \in \mathbb{R}$. Then with probability one no two finite paths of any lengths have equal weight. Consequently for any $v \in \mathbb{Z}_{+}^{2}$ there is a unique finite geodesic between 0 and $v$. Together these finite geodesics form the geodesic tree $\mathscr{T}_{0}$ rooted at 0 that spans $\mathbb{Z}_{+}^{2}$. The two subtrees rooted at $e_{1}$ and $e_{2}$ are separated by an up-right path $\varphi=\left(\varphi_{k}\right)_{k \geq 0}$ on the dual lattice $\left(\frac{1}{2}, \frac{1}{2}\right)+\mathbb{Z}_{+}^{2}$ with $\varphi_{0}=\left(\frac{1}{2}, \frac{1}{2}\right)$. The path $\varphi$ is called the competition interface. The term comes from the interpretation that the subtrees are two competing infections on the lattice $[19,20]$. See Fig. 1.

Adopt the convention that $G_{e_{i}, n e_{j}}=-\infty$ for $i \neq j$ and $n \geq 0$ (there is no admissible path from $e_{i}$ to $n e_{j}$ ). Fix $n \in \mathbb{N}$. As $v$ moves to the right with $|v|_{1}=n$ fixed, the function $G_{e_{2}, v}-G_{e_{1}, v}$ is nonincreasing (Lemma A.2 in the appendix). Then $\varphi_{n-1}=\left(k+\frac{1}{2}, n-k-\frac{1}{2}\right)$ for the unique $0 \leq k<n$ such that

$$
G_{e_{2},(k, n-k)}-G_{e_{1},(k, n-k)}>0>G_{e_{2},(k+1, n-k-1)}-G_{e_{1},(k+1, n-k-1)} .
$$

Theorem 2.6 Assume $\mathbb{P}\left\{\omega_{0} \leq r\right\}$ is continuous in $r$ and that $g_{\mathrm{pp}}$ is differentiable at the endpoints of all its linear segments. Then we have the law of large numbers

$$
\xi_{*}(\omega)=\lim _{n \rightarrow \infty} n^{-1} \varphi_{n}(\omega) \quad \mathbb{P}-\text { a.s. }
$$

The limit $\xi_{*}$ is almost surely an exposed point in ri $\mathscr{U}$ (recall definition (2.3)). For any $\xi \in \operatorname{ri} \mathscr{U}, \mathbb{P}\left(\xi_{*}=\xi\right)>0$ if and only if $\xi \in($ ri $\mathscr{U}) \backslash \mathscr{D}$. Any open interval outside the closed linear segments of $g_{\mathrm{pp}}$ contains $\xi_{*}$ with positive probability.

When $\omega_{0}$ has continuous distribution, $g_{\mathrm{pp}}$ is expected to be strictly concave. Thus the assumption that $g_{\mathrm{pp}}$ is differentiable at the endpoints of its linear segments should really be vacuously true in the theorem. In light of the expectation that $g_{\mathrm{pp}}$ is differentiable, the conjecture for $\xi_{*}$ would be that it has a continuous distribution.

In the exponential case, (2.17) and the explicit distribution of $\xi_{*}$ were given in Theorem 1 of [20]. 
Remark 2.7 Assume that $\mathbb{P}\left\{\omega_{0} \leq r\right\}$ is continuous and that $g_{\mathrm{pp}}$ is either differentiable or strictly concave on ri $\mathscr{U}$ so that no caveats are needed. The minimum in (2.14) with $B=B^{\xi}$ is taken at $j=1$ if $\xi \cdot e_{1}>\xi_{*}\left(T_{x_{i}} \omega\right) \cdot e_{1}$ and at $j=2$ if $\xi \cdot e_{1}<\xi_{*}\left(T_{x_{i}} \omega\right) \cdot e_{1}$. This will become clear from an alternative definition (5.2) of $\xi_{*}$.

The competition interface is a natural direction in which there are two geodesics out of 0 . Nonuniqueness in the random direction $\xi_{*}$ does not violate the almost sure uniqueness in a fixed direction given in Theorem 2.1(iii). For $x \in \mathbb{Z}^{2}$ let $\mathscr{U}_{*}^{x}$ be the random set of directions $\xi \in \mathscr{U}$ such that there are at least two $\mathscr{U} \xi$-directed semiinfinite geodesics out of $x$.

Theorem 2.8 Assume $\mathbb{P}\left\{\omega_{0} \leq r\right\}$ is continuous in $r$. Assume $g_{\mathrm{pp}}$ is differentiable at the endpoints of all its linear segments. The following statements are true with $\mathbb{P}$-probability one and for all $x \in \mathbb{Z}^{2}$.

(i) $\xi_{*}\left(T_{x} \omega\right)$ is the unique direction $\xi$ such that there are at least two $\mathscr{U} \xi$-directed semi-infinite geodesics from $x$ that separate at $x$ and never intersect thereafter.

(ii) $\mathscr{U}_{*}^{x}$ contains all $\xi \in($ ri $\mathscr{U}) \backslash \mathscr{D}$, intersects every open interval outside the closed linear segments of $g_{\mathrm{pp}}$, and is a countably infinite subset of $\left\{\xi_{*}\left(T_{z} \omega\right): z \geq x\right\}$.

In the exponential case Theorem 1(1)-(2) of [14] showed that $\mathscr{U}_{*}^{x}$ is countably infinite and dense.

Theorems 2.6 and 2.8 are proved in Sect. 5. More is actually true. In Sect. 5 we define $\xi_{*}$ on a larger probability space in terms of a priori constructed cocycles, without any assumptions on $g_{\mathrm{pp}}$. Then even without the differentiability assumptions of Theorems 2.6 and 2.8, corners of the limit shape are the atoms of $\xi_{*}$, and there are at least two $\mathscr{U} \xi_{*} \circ T_{x}$-directed semi-infinite geodesics out of $x$ that immediately separate and never intersect after that. (See Theorem 5.3 below.)

When $\omega_{0}$ does not have continuous distribution, there are two competition interfaces: one for the tree of leftmost geodesics and one for the tree of rightmost geodesics. Then $\xi_{*}$ has natural left and right versions, defined in (5.8). We compute the limit distributions of the two competition interfaces for geometric weights in Sects. 2.7 and 6.

\subsection{Exactly solvable models}

We illustrate our results in the two exactly solvable cases: the distribution of the weights $\omega_{x}$ with mean $m_{0}>0$ is either

exponential: $\mathbb{P}\left\{\omega_{x} \geq t\right\}=e^{-t / m_{0}} \quad$ for $t \in \mathbb{R}_{+}$with $\sigma^{2}=m_{0}^{2}$,

or geometric: $\mathbb{P}\left\{\omega_{x} \geq k\right\}=\left(1-m_{0}^{-1}\right)^{k} \quad$ for $k \in \mathbb{N}$ with $\sigma^{2}=m_{0}\left(m_{0}-1\right)$.

For both cases the point-to-point limit function is

$$
g_{\mathrm{pp}}(\xi)=m_{0}\left(\xi \cdot e_{1}+\xi \cdot e_{2}\right)+2 \sigma \sqrt{\left(\xi \cdot e_{1}\right)\left(\xi \cdot e_{2}\right)}
$$

In the exponential case this formula was first derived by Rost [41] (who presented the model in its coupling with TASEP without the last-passage formulation) while early derivations of the geometric case appeared in $[13,30,42]$. 
Since $g_{\text {pp }}$ is differentiable and strictly concave, ri $\mathscr{U}=\mathscr{E}$ and all the results of the previous sections are valid. Theorem 2.3 implies that Busemann functions (2.12) exist in all directions $\xi \in \mathrm{ri} \mathscr{U}$. The probability distribution of $B^{\xi}$ can be described explicitly. For the exponential case see for example Theorem 8.1 in [11] or Section 3.3 in [12], and Sections 3.1 and 7.1 in [23] for both cases.

Section 2.4 gives the following results on geodesics. For almost every $\omega$ every semi-infinite geodesic has a direction. For every fixed direction $\xi \in$ ri $\mathscr{U}$ the following holds almost surely. There exists a $\xi$-geodesic out of every lattice point. In the exponential case, these $\xi$-geodesics are unique and coalesce. In the geometric case uniqueness cannot hold, but there exists a unique leftmost $\xi$-geodesic out of each lattice point and these leftmost $\xi$-geodesics coalesce. The same holds for rightmost $\xi$-geodesics. Finite (leftmost/rightmost) geodesics from $u \in \mathbb{Z}^{2}$ to $v_{n}$ converge to infinite (leftmost/rightmost) $\xi$-geodesics out of $u$, as $v_{n} /\left|v_{n}\right|_{1} \rightarrow \xi$ with $\left|v_{n}\right|_{1} \rightarrow \infty$.

The description of random directions for nonuniqueness of geodesics in Theorem 2.8(i)-(ii) applies to the exponential case. In the exponential case the asymptotic direction $\xi_{*}$ of the competition interface given by Theorem 2.6 has been studied by several authors, not only for percolation in the first quadrant $\mathbb{Z}_{+}^{2}$ as studied here, but with much more general initial profiles [12,19,20].

The model with geometric weights has a tree of leftmost geodesics with competition interface $\varphi^{(l)}=\left(\varphi_{k}^{(l)}\right)_{k \geq 0}$ and a tree of rightmost geodesics with competition interface $\varphi^{(r)}=\left(\varphi_{k}^{(r)}\right)_{k \geq 0}$. Note that $\varphi^{(r)}$ is to the left of $\varphi^{(l)}$ because in (2.16) there is now a middle range $G_{e_{2},(k, n-k)}-G_{e_{1},(k, n-k)}=0$ that is to the right (left) of $\varphi^{(r)}\left(\varphi^{(l)}\right)$. Strict concavity of the limit $g_{\mathrm{pp}}$ implies (with the arguments of Sect. 5) the almost sure limits

$$
n^{-1} \varphi_{n}^{(l)} \rightarrow \xi_{*}^{(l)} \text { and } n^{-1} \varphi_{n}^{(r)} \rightarrow \xi_{*}^{(r)}
$$

The angles $\theta_{*}^{(a)}=\tan ^{-1}\left(\xi_{*}^{(a)} \cdot e_{2} / \xi_{*}^{(a)} \cdot e_{1}\right)$ for $a \in\{l, r\}$ have the following distributions (with $p_{0}=m_{0}^{-1}$ denoting the success probability of the geometric): for $t \in[0, \pi / 2]$

$$
\begin{aligned}
\mathbb{P}\left\{\theta_{*}^{(r)} \leq t\right\} & =\frac{\sqrt{\left(1-p_{0}\right) \sin t}}{\sqrt{\left(1-p_{0}\right) \sin t}+\sqrt{\cos t}} \\
\text { and } \quad \mathbb{P}\left\{\theta_{*}^{(l)} \leq t\right\} & =\frac{\sqrt{\sin t}}{\sqrt{\sin t}+\sqrt{\left(1-p_{0}\right) \cos t}} .
\end{aligned}
$$

Section 6 derives (2.18). Taking $p_{0} \rightarrow 0$ recovers the exponential case first proved in [20].

We turn to describe the setting of stationary cocycles in which our results are proved.

\section{Stationary cocycles and Busemann functions}

The results of this paper are based on a construction of stationary cocycles on an extended space $\widehat{\Omega}=\Omega \times \Omega^{\prime}$ where $\Omega=\mathbb{R}^{\mathbb{Z}^{2}}$ is the original environment space and $\Omega^{\prime}=S^{\mathbb{Z}^{2}}$ is another Polish product space. The details of the construction are in 
Section 7 of [23]. Spatial translations act in the usual manner: with generic elements of $\widehat{\Omega}$ denoted by $\widehat{\omega}=\left(\omega, \omega^{\prime}\right)=\left(\omega_{x}, \omega_{x}^{\prime}\right)_{x \in \mathbb{Z}^{2}}=\left(\widehat{\omega}_{x}\right)_{x \in \mathbb{Z}^{2}},\left(T_{x} \widehat{\omega}\right)_{y}=\widehat{\omega}_{x+y}$ for $x, y \in \mathbb{Z}^{2}$. The extended probability space is $(\widehat{\Omega}, \widehat{\mathfrak{S}}, \widehat{\mathbb{P}})$ where $\widehat{\mathfrak{S}}$ is the Borel $\sigma$ algebra and $\widehat{\mathbb{P}}$ is a translation-invariant probability measure. $\widehat{\mathbb{E}}$ denotes expectation under $\widehat{\mathbb{P}}$. In this setting a cocycle is defined as follows.

Definition 3.1 A measurable function $B: \widehat{\Omega} \times \mathbb{Z}^{2} \times \mathbb{Z}^{2} \rightarrow \mathbb{R}$ is a stationary $L^{1}(\widehat{\mathbb{P}})$ cocycle if it satisfies the following three conditions $\forall x, y, z \in \mathbb{Z}^{2}$.

(a) Integrability: $\widehat{\mathbb{E}}|B(x, y)|<\infty$.

(b) Stationarity: for $\widehat{\mathbb{P}}$-a.e. $\widehat{\omega}, B(\widehat{\omega}, z+x, z+y)=B\left(T_{z} \widehat{\omega}, x, y\right)$.

(c) Additivity: for $\widehat{\mathbb{P}}$-a.e. $\widehat{\omega}, B(\widehat{\omega}, x, y)+B(\widehat{\omega}, y, z)=B(\widehat{\omega}, x, z)$.

The cocycles of interest are related to the last-passage weights through the next definition.

Definition 3.2 A stationary $L^{1}$ cocycle $B$ on $\widehat{\Omega}$ recovers weights $\omega$ if

$$
\omega_{x}=\min _{i \in\{1,2\}} B\left(\widehat{\omega}, x, x+e_{i}\right) \text { for } \widehat{\mathbb{P}} \text {-a.e. } \widehat{\omega} \text { and } \forall x \in \mathbb{Z}^{2}
$$

The next theorem (reproduced from Theorem 5.2 in [23]) states the existence and properties of the cocycles. Assumption (2.1) is in force. This is the only place where the assumption $\mathbb{P}\left(\omega_{0} \geq c\right)=1$ is needed, and the only reason is that the queueing results that are used to prove the theorem assume $\omega_{0} \geq 0$. In part (i) below we use this notation: for a finite or infinite set $I \subset \mathbb{Z}^{2}, I^{<}=\left\{x \in \mathbb{Z}^{2}: x \geq z \forall z \in I\right\}$ is the set of lattice points that do not lie on a ray from $I$ at an angle in $[0, \pi / 2]$. For example, if $I=\{0, \ldots, m\} \times\{0, \ldots, n\}$ then $I^{<}=\mathbb{Z}^{2} \backslash \mathbb{Z}_{+}^{2}$.

Theorem 3.3 There exist real-valued Borel functions $B_{ \pm}^{\xi}(\widehat{\omega}, x, y)$ of $(\widehat{\omega}, \xi, x, y) \in$ $\widehat{\Omega} \times \operatorname{ri} \mathscr{U} \times \mathbb{Z}^{2} \times \mathbb{Z}^{2}$ and a translation invariant Borel probability measure $\widehat{\mathbb{P}}$ on $(\widehat{\Omega}, \widehat{\mathfrak{S}})$ such that the following properties hold.

(i) Under $\widehat{\mathbb{P}}$, the marginal distribution of the configuration $\omega$ is the i.i.d. measure $\mathbb{P}$ specified in assumption (2.1). For each $\xi \in \mathrm{ri} \mathscr{U}$ and \pm , the $\mathbb{R}^{3}$-valued process $\left\{\psi_{x}^{ \pm, \xi}\right\}_{x \in \mathbb{Z}^{2}}$ defined by

$$
\psi_{x}^{ \pm, \xi}(\widehat{\omega})=\left(\omega_{x}, B_{ \pm}^{\xi}\left(\widehat{\omega}, x, x+e_{1}\right), B_{ \pm}^{\xi}\left(\widehat{\omega}, x, x+e_{2}\right)\right)
$$

is separately ergodic under both translations $T_{e_{1}}$ and $T_{e_{2}}$. For any $I \subset \mathbb{Z}^{2}$, the variables

$$
\left\{\left(\omega_{x}, B_{+}^{\xi}\left(\widehat{\omega}, x, x+e_{i}\right), B_{-}^{\xi}\left(\widehat{\omega}, x, x+e_{i}\right)\right): x \in I, \xi \in \operatorname{ri} \mathscr{U}, i \in\{1,2\}\right\}
$$

are independent of $\left\{\omega_{x}: x \in I^{<}\right\}$. 
(ii) Each process $B_{ \pm}^{\xi}=\left\{B_{ \pm}^{\xi}(x, y)\right\}_{x, y \in \mathbb{Z}^{2}}$ is a stationary $L^{1}(\widehat{\mathbb{P}})$ cocycle (Definition 3.1) that recovers the weights $\omega$ (Definition 3.2):

$$
\omega_{x}=B_{ \pm}^{\xi}\left(\widehat{\omega}, x, x+e_{1}\right) \wedge B_{ \pm}^{\xi}\left(\widehat{\omega}, x, x+e_{2}\right) \quad \widehat{\mathbb{P}}-\text { a.s. }
$$

The mean vectors satisfy

$$
\widehat{\mathbb{E}}\left[B_{ \pm}^{\xi}\left(0, e_{1}\right)\right] e_{1}+\widehat{\mathbb{E}}\left[B_{ \pm}^{\xi}\left(0, e_{2}\right)\right] e_{2}=\nabla g_{\mathrm{pp}}(\xi \pm) .
$$

(iii) No two distinct cocycles have a common mean vector. That is, if $\nabla g_{\mathrm{pp}}(\xi+)$ $=\nabla g_{\mathrm{pp}}(\zeta-)$ then

$$
B_{+}^{\xi}(\widehat{\omega}, x, y)=B_{-}^{\zeta}(\widehat{\omega}, x, y) \quad \forall \widehat{\omega} \in \widehat{\Omega}, x, y \in \mathbb{Z}^{2}
$$

and similarly for all four combinations of \pm and $\xi$, $\zeta$. These equalities hold for all $\widehat{\omega}$ without an almost sure modifier because they come directly from the construction. In particular, if $\xi \in \mathscr{D}$ then

$$
B_{+}^{\xi}(\widehat{\omega}, x, y)=B_{-}^{\xi}(\widehat{\omega}, x, y)=B^{\xi}(\widehat{\omega}, x, y) \quad \forall \widehat{\omega} \in \widehat{\Omega}, x, y \in \mathbb{Z}^{2},
$$

where the second equality defines the cocycle $B^{\xi}$.

(iv) There exists an event $\widehat{\Omega}_{0}$ with $\widehat{\mathbb{P}}\left(\widehat{\Omega}_{0}\right)=1$ and such that (a) and (b) below hold for all $\widehat{\omega} \in \widehat{\Omega}_{0}, x, y \in \mathbb{Z}^{2}$ and $\xi, \zeta \in$ ri $\mathscr{U}$.

(a) Monotonicity: if $\xi \cdot e_{1}<\zeta \cdot e_{1}$ then

$$
\begin{aligned}
B_{-}^{\xi}\left(\widehat{\omega}, x, x+e_{1}\right) & \geq B_{+}^{\xi}\left(\widehat{\omega}, x, x+e_{1}\right) \geq B_{-}^{\zeta}\left(\widehat{\omega}, x, x+e_{1}\right) \\
\text { and } \quad B_{-}^{\xi}\left(\widehat{\omega}, x, x+e_{2}\right) & \leq B_{+}^{\xi}\left(\widehat{\omega}, x, x+e_{2}\right) \leq B_{-}^{\zeta}\left(\widehat{\omega}, x, x+e_{2}\right) .
\end{aligned}
$$

(b) Right continuity: if $\zeta_{n} \cdot e_{1} \searrow \xi \cdot e_{1}$ then

$$
\lim _{n \rightarrow \infty} B_{ \pm}^{\zeta_{n}}(\widehat{\omega}, x, y)=B_{+}^{\xi}(\widehat{\omega}, x, y)
$$

(v) Left continuity at a fixed $\xi \in \mathrm{ri} \mathscr{U}$ : there exists an event $\widehat{\Omega}^{(\xi)}$ with $\widehat{\mathbb{P}}\left(\widehat{\Omega}^{(\xi)}\right)=1$ and such that for any sequence $\zeta_{n} \cdot e_{1} \nearrow \xi \cdot e_{1}$

$$
\lim _{n \rightarrow \infty} B_{ \pm}^{\zeta_{n}}(\widehat{\omega}, x, y)=B_{-}^{\xi}(\widehat{\omega}, x, y) \quad \text { for } \widehat{\omega} \in \widehat{\Omega}^{(\xi)}, x, y \in \mathbb{Z}^{d}
$$

The next result (Theorem 6.1 in [23]) relates the cocycles $B_{ \pm}^{\xi}$ to limiting $G$ increments. We quote the theorem in full for use in the proof of Theorem 2.4 below. (2.1) is assumed. Recall the line segment $\mathscr{U}_{\xi}=[\underline{\xi}, \bar{\xi}]$ with $\underline{\xi} \cdot e_{1} \leq \bar{\xi} \cdot e_{1}$ from (2.6)-(2.7). 
Theorem 3.4 Fix $\xi \in$ ri $\mathscr{U}$. Then there exists an event $\widehat{\Omega}_{0}$ with $\widehat{\mathbb{P}}\left(\widehat{\Omega}_{0}\right)=1$ such that for each $\widehat{\omega} \in \widehat{\Omega}_{0}$ and for any sequence $v_{n} \in \mathbb{Z}_{+}^{2}$ that satisfies

$$
\left|v_{n}\right|_{1} \rightarrow \infty \text { and } \underline{\xi} \cdot e_{1} \leq \varliminf_{n \rightarrow \infty} \frac{v_{n} \cdot e_{1}}{\left|v_{n}\right|_{1}} \leq \varlimsup_{n \rightarrow \infty} \frac{v_{n} \cdot e_{1}}{\left|v_{n}\right|_{1}} \leq \bar{\xi} \cdot e_{1},
$$

we have

$$
\begin{aligned}
B_{+}^{\bar{\xi}}\left(\widehat{\omega}, x, x+e_{1}\right) & \leq \varliminf_{n \rightarrow \infty}\left(G_{x, v_{n}}(\omega)-G_{x+e_{1}, v_{n}}(\omega)\right) \\
& \leq \varlimsup_{n \rightarrow \infty}\left(G_{x, v_{n}}(\omega)-G_{x+e_{1}, v_{n}}(\omega)\right) \leq B_{-}^{\xi}\left(\widehat{\omega}, x, x+e_{1}\right)
\end{aligned}
$$

and

$$
\begin{aligned}
B_{-}^{\underline{\xi}}\left(\widehat{\omega}, x, x+e_{2}\right) & \leq \varliminf_{n \rightarrow \infty}\left(G_{x, v_{n}}(\omega)-G_{x+e_{2}, v_{n}}(\omega)\right) \\
& \leq \varlimsup_{n \rightarrow \infty}\left(G_{x, v_{n}}(\omega)-G_{x+e_{2}, v_{n}}(\omega)\right) \leq B_{+}^{\bar{\xi}}\left(\widehat{\omega}, x, x+e_{2}\right) .
\end{aligned}
$$

Remark 3.5 (i) Theorem 2.3 follows immediately because by Theorem 3.3(iii), $B_{ \pm}^{\xi}=$ $B^{\xi}=B_{ \pm}^{\bar{\xi}}$ if $\xi, \underline{\xi}, \bar{\xi} \in \mathscr{D}$. (ii) If $g_{\mathrm{pp}}$ is assumed differentiable at the endpoints of all its linear segments, then all cocycles $B_{ \pm}^{\xi}(x, y)$ are in fact functions of $\omega$, that is, S-measurable (see Theorem 5.3 in [23]).

\section{Directional geodesics}

This section proves the results on geodesics. We define special geodesics in terms of the cocycles $B_{ \pm}^{\xi}$ from Theorem 3.3, on the extended space $\widehat{\Omega}=\Omega \times \Omega^{\prime}$. Assumption (2.1) is in force. The idea is in the next lemma, followed by the definition of cocycle geodesics.

Lemma 4.1 Fix $\omega \in \Omega$. Assume a function $B: \mathbb{Z}^{2} \times \mathbb{Z}^{2} \rightarrow \mathbb{R}$ satisfies

$$
\begin{aligned}
& B(x, y)+B(y, z)=B(x, z) \text { and } \omega_{x}=B\left(x, x+e_{1}\right) \wedge B\left(x, x+e_{2}\right) \\
& \forall x, y, z \in \mathbb{Z}^{2} .
\end{aligned}
$$

(a) Let $x_{m, n}=\left(x_{k}\right)_{k=m}^{n}$ be any up-right path that follows minimal gradients of $B$, that is,

$$
\omega_{x_{k}}=B\left(x_{k}, x_{k+1}\right) \quad \text { for all } m \leq k<n .
$$

Then $x_{m, n}$ is a geodesic from $x_{m}$ to $x_{n}$ :

$$
G_{x_{m}, x_{n}}(\omega)=\sum_{k=m}^{n-1} \omega_{x_{k}}=B\left(x_{m}, x_{n}\right)
$$


(b) Let $x_{m, n}=\left(x_{k}\right)_{k=m}^{n}$ be an up-right path such that for all $m \leq k<n$

$$
\begin{aligned}
\text { either } \omega_{x_{k}} & =B\left(x_{k}, x_{k+1}\right)<B\left(x_{k}, x_{k}+e_{1}\right) \vee B\left(x_{k}, x_{k}+e_{2}\right) \\
\text { or } x_{k+1} & =x_{k}+e_{2} \text { and } B\left(x_{k}, x_{k}+e_{1}\right)=B\left(x_{k}, x_{k}+e_{2}\right) .
\end{aligned}
$$

In other words, path $x_{m, n}$ follows minimal gradients of $B$ and takes an $e_{2}$-step in a tie. Then $x_{m, n}$ is the leftmost geodesic from $x_{m}$ to $x_{n}$. Precisely, if $x_{m, n}^{\prime}$ is an up-right path from $x_{m}^{\prime}=x_{m}$ to $x_{n}^{\prime}=x_{n}$ and $G_{x_{m}, x_{n}}=\sum_{k=m}^{n-1} \omega_{x_{k}^{\prime}}$, then $x_{k} \cdot e_{1} \leq x_{k}^{\prime} \cdot e_{1}$ for all $m \leq k \leq n$.

If ties are broken by $e_{1}$-steps the resulting geodesic is the rightmost geodesic between $x_{m}$ and $x_{n}: x_{k} \cdot e_{1} \geq x_{k}^{\prime} \cdot e_{1}$ for all $m \leq k<n$.

Proof Part (a). Any up-right path $y_{m, n}$ from $y_{m}=x_{m}$ to $y_{n}=x_{n}$ satisfies

$$
\sum_{k=m}^{n-1} \omega_{y_{k}} \leq \sum_{k=m}^{n-1} B\left(y_{k}, y_{k+1}\right)=B\left(x_{m}, x_{n}\right)=\sum_{k=m}^{n-1} B\left(x_{k}, x_{k+1}\right)=\sum_{k=m}^{n-1} \omega_{x_{k}} .
$$

Part (b). $x_{m, n}$ is a geodesic by part (a). To prove that it is the leftmost geodesic assume $x_{k}^{\prime}=x_{k}$ and $x_{k+1}=x_{k}+e_{1}$. Then $\omega_{x_{k}}=B\left(x_{k}, x_{k}+e_{1}\right)<B\left(x_{k}, x_{k}+e_{2}\right)$. Recovery of the weights gives $G_{x, y} \leq B(x, y)$ for all $x \leq y$. Combined with (4.1),

$$
\omega_{x_{k}}+G_{x_{k}+e_{2}, x_{n}}<B\left(x_{k}, x_{k}+e_{2}\right)+B\left(x_{k}+e_{2}, x_{n}\right)=B\left(x_{k}, x_{n}\right)=G_{x_{k}, x_{n}} .
$$

Hence also $x_{k+1}^{\prime}=x_{k}^{\prime}+e_{1}$ and the claim about being the leftmost geodesic is proved. The other claim is symmetric.

Next we define cocycle geodesics, that is, geodesics constructed by following minimal gradients of cocycles $B_{ \pm}^{\xi}$ constructed in Theorem 3.3. Since our treatment allows discrete distributions, we introduce a function $\mathfrak{t}$ on $\mathbb{Z}^{2}$ to resolve ties. For $\xi \in \operatorname{ri} \mathscr{U}, u \in \mathbb{Z}^{2}$, and $\mathfrak{t} \in\left\{e_{1}, e_{2}\right\}^{\mathbb{Z}^{2}}$, let $x_{0, \infty}^{u, \mathfrak{t}, \xi, \pm}$ be the up-right path (one path for + , one for -) starting at $x_{0}^{u, \mathfrak{t}, \xi, \pm}=u$ and satisfying for all $n \geq 0$

$$
x_{n+1}^{u, \mathfrak{t}, \xi, \pm}=\left\{\begin{array}{cc}
x_{n}^{u, \mathfrak{t}, \xi, \pm}+e_{1} & \text { if } B_{ \pm}^{\xi}\left(x_{n}^{u, \mathfrak{t}, \xi, \pm}, x_{n}^{u, \mathfrak{t}, \xi, \pm}+e_{1}\right) \\
<B_{ \pm}^{\xi}\left(x_{n}^{u, \mathfrak{t}, \xi, \pm}, x_{n}^{u, \mathfrak{t}, \xi, \pm}+e_{2}\right) \\
x_{n}^{u, \mathfrak{t}, \xi, \pm}+e_{2} & \text { if } B_{ \pm}^{\xi}\left(x_{n}^{u, \mathfrak{t}, \xi, \pm}, x_{n}^{u, \mathfrak{t}, \xi, \pm}+e_{2}\right) \\
<B_{ \pm}^{\xi}\left(x_{n}^{u, \mathfrak{t}, \xi, \pm}, x_{n}^{u, \mathfrak{t}, \xi, \pm}+e_{1}\right) \\
x_{n}^{u, \mathfrak{t}, \xi, \pm}+\mathfrak{t}\left(x_{n}^{u, \mathfrak{t}, \xi, \pm}\right) & \text { if } B_{ \pm}^{\xi}\left(x_{n}^{u, \mathfrak{t}, \xi, \pm}, x_{n}^{u, \mathfrak{t}, \xi, \pm}+e_{1}\right) \\
=B_{ \pm}^{\xi}\left(x_{n}^{u, \mathfrak{t}, \xi, \pm}, x_{n}^{u, \mathfrak{t}, \xi, \pm}+e_{2}\right) .
\end{array}\right.
$$

Cocycles $B_{ \pm}^{\xi}$ satisfy $\omega_{x}=B_{ \pm}^{\xi}\left(\widehat{\omega}, x, x+e_{1}\right) \wedge B_{ \pm}^{\xi}\left(\widehat{\omega}, x, x+e_{2}\right)$ (Theorem 3.3(ii)) and so by Lemma $4.1(\mathrm{a}), x_{0, \infty}^{u, t, \xi}, \pm$ is a semi-infinite geodesic.

Through the cocycles these geodesics are measurable functions on $\widehat{\Omega}$. If $g_{\mathrm{pp}}$ is differentiable at the endpoints of its linear segments (if any), cocycles $B_{ \pm}^{\xi}$ are functions 
of $\omega$ (Theorem 5.3 in [23]). Then geodesics $x_{0, \infty}^{u, \mathfrak{t}, \xi, \pm}$ can be defined on $\Omega$ without the artificial extension to the space $\widehat{\Omega}=\Omega \times \Omega^{\prime}$.

If we restrict ourselves to the event $\widehat{\Omega}_{0}$ of full $\widehat{\mathbb{P}}$-measure on which monotonicity (3.5) holds for all $\xi, \zeta \in \mathrm{ri} \mathscr{U}$, we can order these geodesics in a natural way from left to right. Define a partial ordering on $\left\{e_{1}, e_{2}\right\}^{\mathbb{Z}^{2}}$ by $e_{2} \preceq e_{1}$ and then $\mathfrak{t} \preceq \mathfrak{t}^{\prime}$ coordinatewise. Then on the event $\widehat{\Omega}_{0}$, for any $u \in \mathbb{Z}^{2}, \mathfrak{t} \preceq \mathfrak{t}^{\prime}, \xi, \zeta \in$ ri $\mathscr{U}$ with $\xi \cdot e_{1}<\zeta \cdot e_{1}$, and for all $n \geq 0$,

$$
\begin{array}{r}
x_{n}^{u, \mathfrak{t}, \xi, \pm} \cdot e_{1} \leq x_{n}^{u, \mathfrak{t}^{\prime}, \xi, \pm} \cdot e_{1}, x_{n}^{u, \mathfrak{t}, \xi,-} \cdot e_{1} \leq x_{n}^{u, \mathfrak{t}, \xi,+} \cdot e_{1}, \\
\text { and } x_{n}^{u, \mathfrak{t}, \xi,+} \cdot e_{1} \leq x_{n}^{u, \mathfrak{t}, \zeta,-} \cdot e_{1} .
\end{array}
$$

The leftmost and rightmost tie-breaking rules are $\underline{\mathfrak{t}}(x)=e_{2}$ and $\overline{\mathfrak{t}}(x)=e_{1} \forall x \in \mathbb{Z}^{2}$. The cocycle limits (3.6) and (3.7) force the cocycle geodesics to converge also, as the next lemma shows.

Lemma 4.2 Fix $\xi$ and let $\zeta_{n} \rightarrow \xi$ in ri $\mathscr{U}$. If $\zeta_{n} \cdot e_{1}>\xi \cdot e_{1} \forall n$ then for all $u \in \mathbb{Z}^{2}$

$$
\widehat{\mathbb{P}}\left\{\forall k \geq 0 \exists n_{0}<\infty: n \geq n_{0} \Rightarrow x_{0, k}^{u, \overline{\mathfrak{t}}, \zeta_{n}, \pm}=x_{0, k}^{u, \overline{\mathfrak{t}}, \xi,+}\right\}=1 .
$$

Similarly, if $\zeta_{n} \cdot e_{1} \nearrow \xi \cdot e_{1}$, we have the almost sure coordinatewise limit $x_{0, \infty}^{u, \mathfrak{t}, \zeta_{n}, \pm} \rightarrow$ $x_{0, \infty}^{u, \mathfrak{t}, \xi,-}$.

Proof It is enough to prove the statement for $u=0$. By (3.6) and (3.7), for a given $k$ and large enough $n$, if $x \geq 0$ with $|x|_{1} \leq k$ and $B_{+}^{\xi}\left(x, x+e_{1}\right) \neq B_{+}^{\xi}\left(x, x+e_{2}\right)$, then $B_{ \pm}^{\zeta_{n}}\left(x, x+e_{1}\right)-B_{ \pm}^{\zeta_{n}}\left(x, x+e_{2}\right)$ does not vanish and has the same sign as $B_{+}^{\xi}\left(x, x+e_{1}\right)-B_{+}^{\xi}\left(x, x+e_{2}\right)$. From such $x$ geodesics following the minimal gradient of $B_{ \pm}^{\zeta_{n}}$ or the minimal gradient of $B_{+}^{\xi}$ stay together for their next step. On the other hand, when $B_{+}^{\xi}\left(x, x+e_{1}\right)=B_{+}^{\xi}\left(x, x+e_{2}\right)$, monotonicity (3.5) implies

$$
B_{ \pm}^{\zeta_{n}}\left(x, x+e_{1}\right) \leq B_{+}^{\xi}\left(x, x+e_{1}\right)=B_{+}^{\xi}\left(x, x+e_{2}\right) \leq B_{ \pm}^{\zeta_{n}}\left(x, x+e_{2}\right)
$$

Once again, both the geodesic following the minimal gradient of $B_{ \pm}^{\zeta_{n}}$ and rules $\overline{\mathfrak{t}}$ and the one following the minimal gradients of $B_{+}^{\xi}$ and rules $\overline{\mathfrak{t}}$ will next take the same $e_{1}$-step. This proves (4.3). The other claim is similar.

Recall the segments $\mathscr{U} \xi \mathscr{U} \xi \pm$ in ri $\mathscr{U}$ defined in (2.6)-(2.7) for $\xi \in$ ri $\mathscr{U}$. The next theorem concerns the direction of cocycle geodesics.

Theorem 4.3 There exists an event $\widehat{\Omega}_{0}$ such that $\widehat{\mathbb{P}}\left(\widehat{\Omega}_{0}\right)=1$ and for each $\widehat{\omega} \in \widehat{\Omega}_{0}$ the following holds:

$$
\forall \xi \in \mathrm{ri} \mathscr{U}, \forall \mathfrak{t} \in\left\{e_{1}, e_{2}\right\}^{\mathbb{Z}^{2}}, \forall u \in \mathbb{Z}^{2}: x_{0, \infty}^{u, \mathfrak{t}, \xi, \pm} \text { is } \mathscr{U}_{\xi \pm} \text {-directed }
$$

For $\xi \in \mathscr{D}$ the \pm is immaterial in the statement. 
Proof Fix $\xi \in$ ri $\mathscr{U}$ and abbreviate $x_{n}=x_{n}^{u, \overline{\mathfrak{t}}, \xi,+} . G_{u, x_{n}}=B_{+}^{\xi}\left(u, x_{n}\right)$ by Lemma 4.1(a). Apply Theorem A.1 with cocycle $B_{+}^{\xi}$ to write

$$
\lim _{n \rightarrow \infty}\left|x_{n}\right|_{1}^{-1}\left(G_{u, x_{n}}-\nabla g_{\mathrm{pp}}(\xi+) \cdot x_{n}\right)=0 \quad \widehat{\mathbb{P}} \text {-almost surely. }
$$

Define $\zeta(\widehat{\omega}) \in \mathscr{U}$ by $\zeta \cdot e_{1}=\varlimsup \frac{x_{n} \cdot e_{1}}{\left|x_{n}\right|_{1}}$. If $\zeta \cdot e_{1}>\bar{\xi} \cdot e_{1}$ then $\zeta \notin \mathscr{U} \xi+$ and hence

$$
g_{\mathrm{pp}}(\zeta)-\nabla g_{\mathrm{pp}}(\xi+) \cdot \zeta<g_{\mathrm{pp}}(\xi)-\nabla g_{\mathrm{pp}}(\xi+) \cdot \xi=0
$$

(The equality is from (2.5). For the inequality, concavity gives $\leq$ and $\zeta \notin \mathscr{U} \xi+$ rules out equality.)

Consequently, by the shape theorem (2.2), on the event $\left\{\zeta \cdot e_{1}>\bar{\xi} \cdot e_{1}\right\}$,

$$
\underline{\lim }_{n \rightarrow \infty}\left|x_{n}\right|_{1}^{-1}\left(G_{u, x_{n}}-\nabla g_{\mathrm{pp}}(\xi+) \cdot x_{n}\right)<0 .
$$

This proves that

$$
\widehat{\mathbb{P}}\left\{\varlimsup_{n \rightarrow \infty} \frac{x_{n}^{u, \overline{\mathrm{t}}, \xi,+} \cdot e_{1}}{\left|x_{n}^{u, \overline{\mathrm{t}}, \xi,+}\right|_{1}} \leq \bar{\xi} \cdot e_{1}\right\}=1
$$

Repeat the same argument with $\overline{\mathfrak{t}}$ replaced by $\underline{\mathfrak{t}}$ and $\bar{\xi}$ by the other endpoint of $\mathscr{U}_{\xi}+$ (which is either $\xi$ or $\underline{\xi}$ ). To capture all $t$ use geodesics ordering (4.2). An analogous argument works for $\xi-$. We have, for a given $\xi$,

$$
\widehat{\mathbb{P}}\left\{\forall \mathfrak{t} \in\left\{e_{1}, e_{2}\right\}^{\mathbb{Z}^{2}}, \forall u \in \mathbb{Z}^{2}: x_{0, \infty}^{u, \mathfrak{t}, \xi, \pm} \text { is } \mathscr{U}_{\xi \pm} \text {-directed }\right\}=1
$$

Let $\widehat{\Omega}_{0}$ be an event of full $\widehat{\mathbb{P}}$-probability on which all cocycle geodesics satisfy the ordering (4.2), and the event in (4.5) holds for both + and - and for $\xi$ in a countable set $\mathscr{U}_{0}$ that contains all points of nondifferentiability of $g_{\text {pp }}$ and a countable dense subset of $\mathscr{D}$. We argue that (4.4) holds on $\widehat{\Omega}_{0}$.

Let $\zeta \notin \mathscr{U}_{0}$ and let $\bar{\zeta}$ denote the right endpoint of $\mathscr{U}_{\zeta}$. We show that

$$
\varlimsup_{n \rightarrow \infty} \frac{x_{n}^{u, \bar{t}, \zeta} \cdot e_{1}}{\left|x_{n}^{u, \bar{t}, \zeta}\right|_{1}} \leq \bar{\zeta} \cdot e_{1} \quad \text { on the event } \widehat{\Omega}_{0}
$$

(Since $\zeta \in \mathscr{D}$ there is no \pm distinction in the cocycle geodesic.) The lim with $\underline{\mathfrak{t}}$ and $\geq \underline{\zeta} \cdot e_{1}$ comes of course with the same argument.

If $\zeta \cdot e_{1}<\bar{\zeta} \cdot e_{1}$ pick $\xi \in \mathscr{D} \cap \mathscr{U}_{0}$ so that $\zeta \cdot e_{1}<\xi \cdot e_{1}<\bar{\zeta} \cdot e_{1}$. Then $\bar{\xi}=\bar{\zeta}$ and (4.6) follows from the ordering.

If $\zeta=\bar{\zeta}$, let $\varepsilon>0$ and pick $\xi \in \mathscr{D} \cap \mathscr{U}_{0}$ so that $\zeta \cdot e_{1}<\xi \cdot e_{1} \leq \bar{\xi} \cdot e_{1}<\zeta \cdot e_{1}+\varepsilon$. This is possible because $\nabla g_{\mathrm{pp}}(\xi)$ converges to but never equals $\nabla g_{\mathrm{pp}}(\zeta)$ as $\xi \cdot e_{1} \searrow \zeta \cdot e_{1}$. Again by the ordering 


$$
\varlimsup_{n \rightarrow \infty} \frac{x_{n}^{u, \bar{t}, \zeta} \cdot e_{1}}{\left|x_{n}^{u, \bar{t}, \zeta}\right|_{1}} \leq \varlimsup_{n \rightarrow \infty} \frac{x_{n}^{u, \bar{t}, \xi} \cdot e_{1}}{\left|x_{n}^{u, \bar{t}, \xi}\right|_{1}} \leq \bar{\xi} \cdot e_{1}<\zeta \cdot e_{1}+\varepsilon .
$$

This completes the proof of Theorem 4.3.

Lemma 4.4 (a) Fix $\xi \in$ ri $\mathscr{U}$. Then the following holds $\widehat{\mathbb{P}}$-almost surely. For any semi-infinite geodesic $x_{0, \infty}$

$$
\varlimsup_{n \rightarrow \infty} \frac{x_{n} \cdot e_{1}}{\left|x_{n}\right|_{1}} \geq \xi \cdot e_{1} \quad \text { implies that } x_{n} \cdot e_{1} \geq x_{n}^{x_{0}, \underline{\mathfrak{t}}, \underline{\xi},-} \cdot e_{1} \text { for all } n \geq 0
$$

and

$$
\varliminf_{n \rightarrow \infty} \frac{x_{n} \cdot e_{1}}{\left|x_{n}\right|_{1}} \leq \xi \cdot e_{1} \quad \text { implies that } x_{n} \cdot e_{1} \leq x_{n}^{x_{0}, \overline{\mathfrak{t}}, \bar{\xi},+} \cdot e_{1} \text { for all } n \geq 0 \text {. }
$$

(b) Fix a maximal line segment $[\underline{\xi}, \bar{\xi}]$ on which $g_{\mathrm{pp}}$ is linear and such that $\underline{\xi} \cdot e_{1}<\bar{\xi} \cdot e_{1}$. Assume $\underline{\xi}, \bar{\xi} \in \mathscr{D}$. Then the following statement holds $\widehat{\mathbb{P}}$-almost surely. Any semiinfinite geodesic $x_{0, \infty}$ such that a limit point of $x_{n} /\left|x_{n}\right|_{1}$ lies in $[\underline{\xi}, \bar{\xi}]$ satisfies

$$
x_{n}^{x_{0}, \underline{t}, \underline{\xi}} \cdot e_{1} \leq x_{n} \cdot e_{1} \leq x_{n}^{x_{0}, \bar{t}, \bar{\xi}} \cdot e_{1} \text { for all } n \geq 0
$$

Proof Part (a). We prove (4.7). (4.8) is proved similarly. Fix a sequence $\zeta_{\ell} \in \mathscr{D}$ such that $\zeta_{\ell} \cdot e_{1} \nearrow \underline{\xi} \cdot e_{1}$ so that, in particular, $\xi \notin \mathscr{U}_{\zeta_{\ell}}$. The good event of full $\widehat{\mathbb{P}}$-probability is the one on which $x_{0, \infty}^{x_{0}, \mathfrak{t}, \zeta_{\ell}}$ is $\mathscr{U}_{\zeta \ell}$-directed (Theorem 4.3), $x_{0, \infty}^{x_{0}, \mathfrak{t}, \zeta_{\ell}}$ is the leftmost geodesic between any two of its points (Lemma 4.1(b) applied to cocycle $B^{\zeta_{\ell}}$ ) and $x_{0, \infty}^{x_{0}, \mathfrak{t}, \zeta_{\ell}} \rightarrow x_{0, \infty}^{x_{0}, \underline{t}, \underline{\xi},-}$ (Lemma 4.2).

By the leftmost property, if $x_{0, \infty}^{x_{0}, \underline{t}, \zeta_{\ell}}$ ever goes strictly to the right of $x_{0, \infty}$, these two geodesics cannot touch again at any later time. But by virtue of the limit points, $x_{n}^{x_{0}, \mathfrak{t}, \zeta \ell} \cdot e_{1}<x_{n} \cdot e_{1}$ for infinitely many $n$. Hence $x_{0, \infty}^{x_{0}, \mathfrak{t}, \zeta \ell}$ stays weakly to the left of $x_{0, \infty}$. Let $\ell \rightarrow \infty$.

Part (b) is proved similarly. The differentiability assumption implies that the geodesic $x_{0, \infty}^{x_{0}, \underline{t}, \underline{\xi}}$ can be approached from the left by geodesics $x_{0, \infty}^{x_{0}, \underline{t}, \zeta_{\ell}}$ such that $\underline{\xi} \notin \mathscr{U}_{\zeta_{\ell}}$.

The next result concerns coalescence of cocycle geodesics $\left\{x_{0, \infty}^{u, \mathfrak{t}, \xi, \pm}: u \in \mathbb{Z}^{2}\right\}$ for fixed $\mathfrak{t}, \pm$, and $\xi \in$ ri $\mathscr{U}$. We can consider a random, stationary tie-breaking function $\mathfrak{t}: \widehat{\Omega} \times \mathbb{Z}^{2} \rightarrow\left\{e_{1}, e_{2}\right\}$ that satisfies

$$
\mathfrak{t}(\widehat{\omega}, x)=\mathfrak{t}\left(T_{x} \widehat{\omega}, 0\right) \quad \forall x \in \mathbb{Z}^{2}, \widehat{\mathbb{P}} \text {-almost surely }
$$


Theorem 4.5 Fix a tie-breaking function $\mathfrak{t}$ that satisfies (4.10) and fix $\xi \in \mathrm{ri} \mathscr{U}$. Then $\widehat{\mathbb{P}}$-almost surely, for all $u, v \in \mathbb{Z}^{2}$, there exist $n, m \geq 0$ such that $x_{n, \infty}^{u, t, \xi,-}=x_{m, \infty}^{v, \mathfrak{t}, \xi,-}$, with a similar statement for + .

Theorem 4.5 is proved by adapting the argument of [31], originally presented for first passage percolation, and by utilizing the independence property in Theorem 3.3(i). Briefly, the idea is the following. By stationarity the assumption of two nonintersecting geodesics implies we can find at least three. A local modification of the weights turns the middle geodesic of the triple into a geodesic that stays disjoint from all geodesics that emanate from sufficiently far away. By stationarity at least $\delta L^{2}$ such disjoint geodesics emanate from an $L \times L$ square. This gives a contradiction because there are only $2 L$ boundary points for these geodesics to exit through. Details can be found in Appendix A of the arXiv preprint [22] of this paper.

The coalescence result above rules out the existence of doubly infinite cocycle geodesics (a.s. for a given cocycle). The following theorem gives the rigorous statement. Its proof is given at the end of the section and is based again on a lack-of-space argument, similar to the proof of Theorem 6.9 in [15].

Theorem 4.6 Let $\mathfrak{t}$ be a stationary tie-breaker as in (4.10) and $\xi \in$ ri $\mathscr{U}$. Then $\widehat{\mathbb{P}}$ almost surely, for all $u \in \mathbb{Z}^{2}$, there exist at most finitely many $v \in \mathbb{Z}^{2}$ such that $x_{0, \infty}^{v, \mathfrak{t}, \xi,-}$ goes through $u$. The same statement holds for + .

It is known that, in general, uniqueness of geodesics cannot hold simultaneously for all directions. In our development this is a consequence of Theorem 5.3 below. As a step towards uniqueness of geodesics in a given direction, the next lemma shows that continuity of the distribution of $\omega_{0}$ prevents ties in (3.3). (The construction of the cocycles implies, through equation (7.6) in [23], that variables $B_{ \pm}^{\xi}\left(x, x+e_{i}\right)$ have continuous marginal distributions. Here we need a property of the joint distribution.) Consequently, for a given $\xi$, $\widehat{\mathbb{P}}$-almost surely geodesics $x_{0, \infty}^{u, \mathfrak{t}, \xi, \pm}$ do not depend on $\mathfrak{t}$.

Lemma 4.7 Assume that $\mathbb{P}\left\{\omega_{0} \leq r\right\}$ is a continuous function of $r \in \mathbb{R}$. Fix $\xi \in$ ri $\mathscr{U}$. Then for all $u \in \mathbb{Z}^{2}$,

$$
\widehat{\mathbb{P}}\left\{B_{+}^{\xi}\left(u, u+e_{1}\right)=B_{+}^{\xi}\left(u, u+e_{2}\right)\right\}=\widehat{\mathbb{P}}\left\{B_{-}^{\xi}\left(u, u+e_{1}\right)=B_{-}^{\xi}\left(u, u+e_{2}\right)\right\}=0 .
$$

Proof Due to shift invariance it is enough to prove the claim for $u=0$. We work with the + case, the other case being similar.

Assume by way of contradiction that the probability in question is positive. By Theorem $4.5, x_{0, \infty}^{e_{2}, \overline{\mathrm{t}}, \xi,+}$ and $x_{0, \infty}^{e_{1}, \overline{\mathrm{t}}, \xi,+}$ coalesce with probability one. Hence there exists $v \in \mathbb{Z}^{2}$ and $n \geq 1$ such that

$$
\mathbb{P}\left\{B_{+}^{\xi}\left(0, e_{1}\right)=B_{+}^{\xi}\left(0, e_{2}\right), x_{n}^{e_{1}, \overline{\mathfrak{t}}, \xi,+}=x_{n}^{e_{2}, \overline{\mathfrak{t}}, \xi,+}=v\right\}>0
$$

Note that if $B_{+}^{\xi}\left(0, e_{1}\right)=B_{+}^{\xi}\left(0, e_{2}\right)$ then both are equal to $\omega_{0}$. Furthermore, by Lemma 4.1(a) we have 


$$
B_{+}^{\xi}\left(e_{1}, v\right)=\sum_{k=0}^{n-1} \omega\left(x_{k}^{e_{1}, \overline{\mathfrak{t}}, \xi,+}\right) \quad \text { and } \quad B_{+}^{\xi}\left(e_{2}, v\right)=\sum_{k=0}^{n-1} \omega\left(x_{k}^{e_{2}, \overline{\mathfrak{t}}, \xi,+}\right) .
$$

(For aesthetic reasons we wrote $\omega(x)$ instead of $\omega_{x}$.) Thus

$$
\begin{aligned}
\omega_{0}+ & \sum_{k=0}^{n-1} \omega\left(x_{k}^{e_{1}, \overline{\mathfrak{t}}, \xi,+}\right)=B_{+}^{\xi}\left(0, e_{1}\right)+B_{+}^{\xi}\left(e_{1}, v\right)=B_{+}^{\xi}(0, v) \\
& =B_{+}^{\xi}\left(0, e_{2}\right)+B_{+}^{\xi}\left(e_{2}, v\right)=\omega_{0}+\sum_{k=0}^{n-1} \omega\left(x_{k}^{e_{2}, \overline{\mathfrak{t}}, \xi,+}\right) .
\end{aligned}
$$

The fact that this happens with positive probability contradicts the assumption that $\omega_{x}$ are i.i.d. and have a continuous distribution.

Proof of Theorem 2.1 Part (i). The existence of $\mathscr{U}_{\xi \pm}$-directed semi-infinite geodesics for $\xi \in$ ri $\mathscr{U}$ follows by fixing $\mathfrak{t}$ and taking geodesics $x_{0, \infty}^{u, \mathfrak{t}, \xi, \pm}$ from Theorem 4.3. For $\xi=e_{i}$ semi-infinite geodesics are simply $x_{0, \infty}=\left(x_{0}+n e_{i}\right)_{n \geq 0}$.

Let $\mathscr{D}_{0}$ be a dense countable subset of $\mathscr{D}$. Let $\widehat{\Omega}_{0}$ be the event of full $\widehat{\mathbb{P}}$-probability on which event (4.4) holds and Lemma 4.4(a) holds for each $u \in \mathbb{Z}^{2}$ and $\zeta \in \mathscr{D}_{0}$. We show that on $\widehat{\Omega}_{0}$, every semi-infinite geodesic is $\mathscr{U}_{\xi}$-directed for some $\xi \in \mathscr{U}$.

Fix $\widehat{\omega} \in \widehat{\Omega}_{0}$ and an arbitrary semi-infinite geodesic $x_{0, \infty}$. Define $\xi^{\prime} \in \mathscr{U}$ by

$$
\xi^{\prime} \cdot e_{1}=\varlimsup_{n \rightarrow \infty} \frac{x_{n} \cdot e_{1}}{\left|x_{n}\right|_{1}}
$$

Let $\xi=\xi^{\prime}=$ the left endpoint of $\mathscr{U}_{\xi^{\prime}}$. We claim that $x_{0, \infty}$ is $\mathscr{U}_{\xi}=[\xi, \bar{\xi}]$-directed. If $\xi^{\prime}=e_{2}$ then $x_{n} /\left|x_{n}\right|_{1} \rightarrow e_{2}$ and $\mathscr{U}_{\xi}=\left\{e_{2}\right\}$ and the case is closed. Suppose $\xi^{\prime} \neq e_{2}$.

The definition of $\xi$ implies that $\xi^{\prime} \in \mathscr{U} \xi+$ and so

$$
\varlimsup_{n \rightarrow \infty} \frac{x_{n} \cdot e_{1}}{\left|x_{n}\right|_{1}}=\xi^{\prime} \cdot e_{1} \leq \bar{\xi} \cdot e_{1}
$$

From the other direction, for any $\zeta \in \mathscr{D}_{0}$ such that $\zeta \cdot e_{1}<\xi^{\prime} \cdot e_{1}$ we have

$$
\varlimsup_{n \rightarrow \infty} \frac{x_{n} \cdot e_{1}}{\left|x_{n}\right|_{1}}>\zeta \cdot e_{1}
$$

which by (4.7) implies $x_{n} \cdot e_{1} \geq x_{n}^{x_{0}, \underline{t}, \underline{\zeta}} \cdot e_{1}$. Then by (4.4)

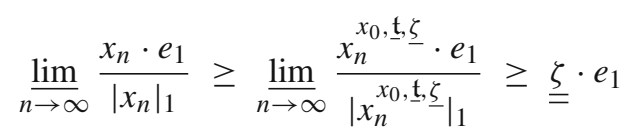

where $\underline{\zeta}=$ the left endpoint of $\mathscr{U}_{\underline{\zeta}}$. It remains to observe that we can take $\underline{\zeta} \cdot e_{1}$ arbitrarily close to $\underline{\bar{\xi}} \cdot e_{1}$. If $\xi \cdot e_{1}<\xi^{\prime} \cdot e_{1}$ then we take $\xi \cdot e_{1}<\zeta \cdot e_{1}<\overline{\overline{\xi^{\prime}}} \cdot e_{1}$ 
in which case $\underline{\zeta}=\xi$ and $\underline{\zeta}=\underline{\xi}$. If $\xi=\xi^{\prime}$ then also $\underline{\xi}=\underline{\xi}^{\prime}=\xi$. In this case, as $\mathscr{D}_{0} \ni \zeta \nearrow \xi, \nabla g_{\mathrm{pp}}(\zeta)$ approaches but never equals $\nabla g_{\mathrm{pp}}(\xi-)$ because there is no flat segment of $g_{\mathrm{pp}}$ adjacent to $\xi$ on the left. This forces both $\underline{\zeta}$ and $\underline{\zeta}$ to converge to $\xi$.

Part (ii). If $g_{\text {pp }}$ is strictly concave then $\mathscr{U} \xi=\{\xi\}$ for all $\xi \in \overline{\overline{\mathrm{r}}} \mathscr{U}$ and (i) $\Rightarrow$ (ii).

Part (iii). By Theorem 3.3(iii) there is a single cocycle $B^{\zeta}$ simultaneously for all $\zeta \in[\underline{\xi}, \bar{\xi}]$. Consequently cocycle geodesics $x_{0, \infty}^{x_{0}, \mathfrak{t}, \underline{\xi}}$ and $x_{0, \infty}^{x_{0}, \mathfrak{t}, \bar{\xi}}$ coincide for any given tie breaking function $\mathfrak{t}$. On the event of full $\mathbb{P}$-probability on which there are no ties between $B^{\zeta}\left(x, x+e_{1}\right)$ and $B^{\zeta}\left(x, x+e_{2}\right)$ the tie breaking function $\mathfrak{t}$ makes no difference. Hence the left and right-hand side of (4.9) coincide. Thus there is no room for two $[\xi, \bar{\xi}]$-directed geodesics from any point. Coalescence comes from Theorem 4.5. The statement about the finite number of ancestors of a site $u$ comes from Theorem 4.6.

Proof of Theorem 2.4 By Theorem 3.4 limit $B$ from (2.12) is now the cocycle $B^{\xi}$. Part (i) follows from Lemma 4.1.

Part (ii). Take sequences $\eta_{n}, \zeta_{n} \in$ ri $\mathscr{U}$ with $\eta_{n} \cdot e_{1}<\underline{\xi} \cdot e_{1} \leq \bar{\xi} \cdot e_{1}<\zeta_{n} \cdot e_{1}$ and $\zeta_{n} \rightarrow \bar{\xi}, \eta_{n} \rightarrow \xi$. Consider the full measure event on which Theorem 3.4 holds for each $\zeta_{n}$ and $\eta_{n}$ with sequences $v_{m}=\left\lfloor m \zeta_{n}\right\rfloor$ and $\left\lfloor m \eta_{n}\right\rfloor$, and on which continuity (3.6) and (3.7) holds as $\zeta_{n} \rightarrow \bar{\xi}, \eta_{n} \rightarrow \xi$. In the rest of the proof we drop the index $n$ from $\zeta_{n}$ and $\eta_{n}$.

We prove the case of a semi-infinite geodesic $x_{0, \infty}$ that satisfies $x_{0}=0$ and (2.15). For large $m,\left\lfloor m \eta \cdot e_{1}\right\rfloor<x_{m} \cdot e_{1}<\left\lfloor m \zeta \cdot e_{1}\right\rfloor$.

Consider first the case $x_{1}=e_{1}$. If there exists a geodesic from 0 to $\lfloor m \zeta\rfloor$ that goes through $e_{2}$, then this geodesic would intersect $x_{0, \infty}$ and thus there would exist another geodesic that goes from 0 to $\lfloor m \zeta\rfloor$ passing through $e_{1}$. In this case we would have $G_{e_{1},\lfloor m \zeta\rfloor}=G_{e_{2},\lfloor m \zeta\rfloor}$. On the other hand, if there exists a geodesic from 0 to $\lfloor m \zeta\rfloor$ that goes through $e_{1}$, then we would have $G_{e_{1},\lfloor m \zeta\rfloor} \geq G_{e_{2},\lfloor m \zeta\rfloor}$. Thus, in either case, we have

$$
G_{0,\lfloor m \zeta\rfloor}-G_{e_{1},\lfloor m \zeta\rfloor} \leq G_{0,\lfloor m \zeta\rfloor}-G_{e_{2},\lfloor m \zeta\rfloor}
$$

Taking $m \rightarrow \infty$ and applying Theorem 3.4 we have $B_{+}^{\bar{\zeta}}\left(0, e_{1}\right) \leq B_{+}^{\bar{\zeta}}\left(0, e_{2}\right)$. Taking $\zeta \rightarrow \bar{\xi}$ and applying (3.6) we have $B_{+}^{\bar{\xi}}\left(0, e_{1}\right) \leq B_{+}^{\bar{\xi}}\left(0, e_{2}\right)$. Since $\bar{\xi}$ and $\xi$ are points of differentiability of $g_{\mathrm{pp}}$, we have $B_{+}^{\bar{\xi}}=B^{\xi}$. Consequently, we have shown $B^{\xi}\left(0, e_{1}\right) \leq$ $B^{\xi}\left(0, e_{2}\right)$. Since $B^{\xi}$ recovers the weights (3.3), the first step $x_{1}=e_{1}$ of $x_{0, \infty}$ satisfies $\omega_{0}=B^{\xi}\left(0, e_{1}\right) \wedge B^{\xi}\left(0, e_{2}\right)=B^{\xi}\left(0, x_{1}\right)$.

When $x_{1}=e_{2}$ repeat the same argument with $\eta$ in place of $\zeta$ to get $B^{\xi}\left(0, e_{2}\right) \leq$ $B^{\xi}\left(0, e_{1}\right)$. This proves the theorem for the first step of the geodesic and that is enough.

Part (iii). We prove the case $i=0$. The statement holds if $B^{\xi}\left(0, e_{1}\right)=B^{\xi}\left(0, e_{2}\right)$, since then both are equal to $\omega_{0}$ by recovery (3.1). If $\omega_{0}=B^{\xi}\left(0, e_{1}\right)<B^{\xi}\left(0, e_{2}\right)$ then convergence (2.12) implies that for $n$ large enough $G_{e_{1}, v_{n}}>G_{e_{2}, v_{n}}$. In this case any maximizing path from 0 to $v_{n}$ will have to start with an $e_{1}$-step and the claim is again true. The case $B^{\xi}\left(0, e_{1}\right)>B^{\xi}\left(0, e_{2}\right)$ is similar. 
Proof of Theorem 2.2 Part (i). $\underline{\underline{\xi}}=\underline{\xi}$ implies $\mathscr{U}_{\underline{\xi}}-\subset \mathscr{U}_{\xi}$ - and, by Theorem 4.3,

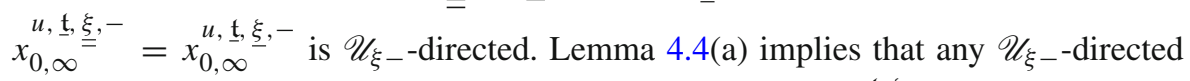
semi-infinite geodesic out of $u \in \mathbb{Z}^{2}$ stays to the right of $x_{0, \infty}^{u, \underline{\mathfrak{t}}, \underline{\underline{\xi}},-}$. Thus $x_{0, \infty}^{u, \underline{\mathfrak{t}}, \underline{\underline{\xi},-}}$ is the leftmost $\mathscr{U}_{\xi}$--directed geodesic out of $u$. The coalescence claim follows now from Theorem 4.5 and the statement about the finite number of ancestors of a site $u$ comes from Theorem 4.6. The case $\overline{\bar{\xi}}=\bar{\xi}$ is similar. Part (i) is proved.

Part (ii). It is enough to work with the case $u=0$. The differentiability assumption implies $B_{ \pm}^{\xi}=B^{\xi}$. We will thus omit the \pm from the $B^{\xi}$-geodesics notation. Take $v_{n}$ as in (2.9). Consider an up-right path $y_{0, \infty}$ that is a limit point of the sequence of leftmost geodesics from 0 to $v_{n}$. By this we mean that along this subsequence, for any $m \in \mathbb{N}$ the initial $m$-step segment of the leftmost geodesic from 0 to $v_{n}$ equals $y_{0, m}$ for $n$ large enough. By Theorem 2.4(iii) we have almost surely $B^{\xi}\left(y_{i}, y_{i+1}\right)=\omega_{y_{i}}$ for all $i \geq 0$. Furthermore, for any $n \in \mathbb{N}, y_{0, n}$ is the leftmost geodesic between 0 and $y_{n}$. We will next show that whenever $B^{\xi}\left(y_{i}, y_{i}+e_{1}\right)=B^{\xi}\left(y_{i}, y_{i}+e_{2}\right)$ we have $y_{i+1}=e_{2}$. This then implies that $y_{0, \infty}=x_{0, \infty}^{0, \mathfrak{t}, \xi}$ and proves part (ii).

It is enough to discuss the case of a tie at $y_{0}=0$. Assume that $B^{\xi}\left(0, e_{1}\right)=B^{\xi}\left(0, e_{2}\right)$ but $y_{1}=e_{1}$. By Theorem 4.5, $x_{0, \infty}^{e_{2}, \overline{\mathfrak{t}}, \xi}$ coalesces with $x_{0, \infty}^{e_{1}, \overline{\mathfrak{t}}, \xi}$. On the other hand, since we already know that $y_{1, \infty}$ follows minimal $B^{\xi}$-gradients we know that it must remain to the left of $x_{0, \infty}^{e_{1}, \bar{t}, \xi}$. This shows that $x_{0, \infty}^{e_{2}, \overline{\mathrm{t}}, \xi}$ intersects $y_{1, \infty}$ at some point $z$ on level $n=|z|_{1}$. But now the path $\bar{y}_{0, n}$ with $\bar{y}_{0}=0$ and $\bar{y}_{1, n}=x_{0, n-1}^{e_{2}, \overline{\mathfrak{t}}, \xi}$ has last passage weights

$$
\omega_{0}+G_{e_{2}, z}=\omega_{0}+B^{\xi}\left(e_{2}, z\right)=B^{\xi}\left(0, e_{2}\right)+B^{\xi}\left(e_{2}, z\right)=B^{\xi}(0, z) \geq G_{0, z}
$$

and is hence a geodesic. (The first equality is because $x_{0, n-1}^{e_{2}, \bar{t}, \xi}$ is a cocycle geodesic, the second comes from weights recovery (3.1) and the tie at 0 , the third is additivity of cocycles, and the last equality is again weights recovery (3.1).) This contradicts the fact that $y_{0, n}$ is the leftmost geodesic from 0 to $y_{n}=z$.

We have thus shown that $y_{0, \infty}=x_{0, \infty}^{0, \mathfrak{t}, \xi}$. A similar statement works for the rightmost geodesics. Part (ii) is proved.

Proof of Theorem 4.6 Let $C_{u}(\widehat{\omega})=\left\{v \in \mathbb{Z}^{2}: x_{0, \infty}^{v, \mathfrak{t}, \xi,-}\right.$ goes through $\left.u\right\}$. The goal is $\widehat{\mathbb{P}}\left\{\left|C_{u}\right|=\infty\right\}=0$. Assume the contrary. Since $C_{u}$ is determined by the ergodic processes (3.2), there is then a positive density of points $u \in \mathbb{Z}^{2}$ with $\left|C_{u}\right|=\infty$.

Consider the tree $\mathscr{G}$ made out of the union of geodesics $x_{0, \infty}^{x, \mathfrak{t}, \xi,-}$ for $x \in \mathbb{Z}^{2}$. (The graph is a tree because once geodesics intersect they merge. It is connected due to coalescence given by Theorem 4.5.) Given $u_{1}, u_{2} \in \mathbb{Z}^{2}$ with $\left|C_{u_{1}}\right|=\left|C_{u_{2}}\right|=\infty$ consider the point where $x_{0, \infty}^{u_{1}, \mathfrak{t}, \xi,-}$ and $x_{0, \infty}^{u_{2}, \mathfrak{t}, \xi,-}$ coalesce. Removing this point from the tree splits the tree into three infinite components. Call such a point a junction point. At each junction point $u$ two infinite admissible paths meet for the first time at $u$, and each path, as a cocycle geodesic, follows the minimal gradients of $B_{-}^{\xi}$ and uses tie-breaking rule $t$. We call these the backward geodesics associated to $u$. 
By shift-invariance and the argument above we have for all $u \in \mathbb{Z}^{2}$

$$
\widehat{\mathbb{P}}\{u \text { is a junction point }\}=\widehat{\mathbb{P}}\{0 \text { is a junction point }\}>0 \text {. }
$$

Then the ergodic theorem implies that there is a positive density of junction points in $\mathbb{Z}^{2}$. We give a lack of space argument that contradicts this.

Let $J$ be the set of junction points in the box $[1, L]^{2}$ together with those points on the south and west boundaries $\left\{k e_{i}: 1 \leq k \leq L, i=1,2\right\}$ where a backward geodesic from a junction point first hits the boundary. Decompose $J$ into finite binary trees by declaring that the two immediate descendants of a junction point are the two closest points on its two backward geodesics that are members of $J$. Then the leaves of these trees are exactly the points on the boundary and the junction points are interior points of the trees. A finite binary tree has more leaves than interior points. Consequently, there cannot be more than $2 L+1$ junction points inside $[1, L]^{2}$. This contradicts (4.11) and proves the theorem.

\section{Competition interface}

This section proves the results of Sect. 2.6. As before, we begin by studying the situation on the extended space $\widehat{\Omega}$ with the help of the cocycles $B_{ \pm}^{\zeta}$ of Theorem 3.3.

Lemma 5.1 Define $B_{-}^{e_{1}}$ and $B_{+}^{e_{2}}$ as the monotone limits of $B_{ \pm}^{\zeta}$ when $\zeta \rightarrow e_{i}, i=1,2$ respectively. Then $\widehat{\mathbb{P}}$-almost surely $B_{-}^{e_{1}}\left(0, e_{1}\right)=B_{+}^{e_{2}}\left(0, e_{2}\right)=\omega_{0}$ and $B_{-}^{e_{1}}\left(0, e_{2}\right)=$ $B_{+}^{e_{2}}\left(0, e_{1}\right)=\infty$.

Proof The limits exist due to monotonicity (3.5). By (3.3) $B_{-}^{e_{1}}\left(0, e_{1}\right) \geq \omega_{0}$ almost surely. Dominated convergence and (3.4) give the limit

$$
\widehat{\mathbb{E}}\left[B_{-}^{e_{1}}\left(0, e_{1}\right)\right]=\lim _{\zeta \rightarrow e_{1}} \widehat{\mathbb{E}}\left[B_{ \pm}^{\zeta}\left(0, e_{1}\right)\right]=\lim _{\zeta \rightarrow e_{1}} e_{1} \cdot \nabla g_{\mathrm{pp}}(\zeta \pm)=\widehat{\mathbb{E}}\left[\omega_{0}\right]
$$

The last equality is a consequence of (2.8) (see Lemma 4.1 and equations (4.12)-(4.13) in [23]). Now $B_{-}^{e_{1}}\left(0, e_{1}\right)=\omega_{0}$ almost surely.

Additivity (Definition 3.1(c)) and recovery (3.3) are satisfied by $B_{-}^{e_{1}}$ and imply

$$
\begin{aligned}
& B_{-}^{e_{1}}\left(n e_{1}, n e_{1}+e_{2}\right) \\
& \quad=\omega_{n e_{1}}+\left(B_{-}^{e_{1}}\left((n+1) e_{1},(n+1) e_{1}+e_{2}\right)-B_{-}^{e_{1}}\left(n e_{1}+e_{2},(n+1) e_{1}+e_{2}\right)\right)^{+} \\
& \quad=\omega_{n e_{1}}+\left(B_{-}^{e_{1}}\left((n+1) e_{1},(n+1) e_{1}+e_{2}\right)-\omega_{n e_{1}+e_{2}}\right)^{+} .
\end{aligned}
$$

The second equality is from the just proved identity $B_{-}^{e_{1}}\left(x, x+e_{1}\right)=\omega_{x}$.

Repeatedly dropping the outer +-part and applying the same formula inductively leads to

$$
\begin{aligned}
B_{-}^{e_{1}}\left(0, e_{2}\right) \geq & \omega_{0}+\sum_{i=1}^{n}\left(\omega_{i e_{1}}-\omega_{(i-1) e_{1}+e_{2}}\right) \\
& +\left(B_{-}^{e_{1}}\left((n+1) e_{1},(n+1) e_{1}+e_{2}\right)-\omega_{n e_{1}+e_{2}}\right)^{+} .
\end{aligned}
$$


Since the summands are i.i.d. with mean 0 , taking $n \rightarrow \infty$ gives $B_{-}^{e_{1}}\left(0, e_{2}\right)=\infty$ almost surely.

Lemma 5.2 Fix $\xi \in($ ri $\mathscr{U}) \backslash \mathscr{D}$ and a tie-breaker $\mathfrak{t}$ that satisfies (4.10). With $\widehat{\mathbb{P}}$-probability one, for any $u \in \mathbb{Z}^{2}$ geodesics $x_{0, \infty}^{u, \mathfrak{t}, \xi,+}$ and $x_{0, \infty}^{u, \mathfrak{t}, \xi,-}$ eventually separate.

Proof Let $A_{u}=\left\{x_{0, \infty}^{u, \mathfrak{t}, \xi,+}=x_{0, \infty}^{u, \mathfrak{t}, \xi,-}\right\}$. We want $\widehat{\mathbb{P}}\left(A_{0}\right)=0$. Assume the contrary. Fix $\zeta \in$ ri $\mathscr{U} . \widehat{\mathbb{P}}\left(A_{0}\right)>0$ and stationarity imply that with positive probability there exists a random sequence $u_{n}=\left\lfloor k_{n} \zeta\right\rfloor$ such that $k_{n} \rightarrow \infty$ and $A_{u_{n}}$ holds for each $n$. Furthermore, for each such $u_{n}$ we know from Theorem 4.5 that $x_{0, \infty}^{u_{0}, \mathfrak{t}, \xi,+}=x_{0, \infty}^{u_{0}, \mathrm{t}, \xi,-}$ and $x_{0, \infty}^{u_{n}, \mathfrak{t}, \xi,+}=x_{0, \infty}^{u_{n}, \mathfrak{t}, \xi,-}$ coalesce at some random point $z_{n}$. By the additivity and (4.1) we then have

$$
\begin{aligned}
B_{+}^{\xi}\left(u_{0}, u_{n}\right) & =B_{+}^{\xi}\left(u_{0}, z_{n}\right)-B_{+}^{\xi}\left(u_{n}, z_{n}\right)=G_{u_{0}, z_{n}}-G_{u_{n}, z_{n}} \\
& =B_{-}^{\xi}\left(u_{0}, z_{n}\right)-B_{-}^{\xi}\left(u_{n}, z_{n}\right)=B_{-}^{\xi}\left(u_{0}, u_{n}\right) .
\end{aligned}
$$

By recovery (3.3) the conditions of Theorem A.1 are satisfied and because of (3.4) we have

$$
\begin{aligned}
& \lim _{n \rightarrow \infty} \frac{B_{+}^{\xi}\left(u_{0}, u_{n}\right)-\nabla g_{\mathrm{pp}}(\xi+) \cdot\left(u_{n}-u_{0}\right)}{\left|u_{n}\right|_{1}} \\
& \quad=0=\lim _{n \rightarrow \infty} \frac{B_{-}^{\xi}\left(u_{0}, u_{n}\right)-\nabla g_{\mathrm{pp}}(\xi-) \cdot\left(u_{n}-u_{0}\right)}{\left|u_{n}\right|_{1}}
\end{aligned}
$$

This and (5.1) lead to $\nabla g_{\mathrm{pp}}(\xi-) \cdot \zeta=\nabla g_{\mathrm{pp}}(\xi+) \cdot \zeta$. Since $\zeta$ is arbitrary we get $\nabla g_{\mathrm{pp}}(\xi-)=\nabla g_{\mathrm{pp}}(\xi+)$, which contradicts the assumption on $\xi$.

Now assume that $\omega_{0}$ has a continuous distribution. By Lemma 4.7 we can omit $\mathfrak{t}$ from the cocycle geodesics notation and write $x_{0, \infty}^{u, \xi, \pm}$.

Next we use the cocycles to define a random variable $\xi_{*}$ on $\widehat{\Omega}$ that represents the asymptotic direction of the competition interface. By Lemma 4.7, with $\widehat{\mathbb{P}}$-probability one, $B_{ \pm}^{\xi}\left(0, e_{1}\right) \neq B_{ \pm}^{\xi}\left(0, e_{2}\right)$ for all rational $\xi \in$ ri $\mathscr{U}$. Furthermore, monotonicity (3.5) gives that

$$
B_{+}^{\zeta}\left(0, e_{1}\right)-B_{+}^{\zeta}\left(0, e_{2}\right) \leq B_{-}^{\zeta}\left(0, e_{1}\right)-B_{-}^{\zeta}\left(0, e_{2}\right) \leq B_{+}^{\eta}\left(0, e_{1}\right)-B_{+}^{\eta}\left(0, e_{2}\right)
$$

when $\zeta \cdot e_{1}>\eta \cdot e_{1}$. Lemma 5.1 implies that $B_{ \pm}^{\zeta}\left(0, e_{1}\right)-B_{ \pm}^{\zeta}\left(0, e_{2}\right)$ converges to $-\infty$ as $\zeta \rightarrow e_{1}$ and to $\infty$ as $\zeta \rightarrow e_{2}$. Thus there exists unique $\xi_{*}(\widehat{\omega}) \in$ ri $\mathscr{U}$ such that for rational $\zeta \in \operatorname{ri} \mathscr{U}$

$$
\text { and } \quad \begin{aligned}
& B_{ \pm}^{\zeta}\left(\widehat{\omega}, 0, e_{1}\right)<B_{ \pm}^{\zeta}\left(\widehat{\omega}, 0, e_{2}\right) \text { if } \zeta \cdot e_{1}>\xi_{*}(\widehat{\omega}) \cdot e_{1} \\
& B_{ \pm}^{\zeta}\left(\widehat{\omega}, 0, e_{1}\right)>B_{ \pm}^{\zeta}\left(\widehat{\omega}, 0, e_{2}\right) \text { if } \zeta \cdot e_{1}<\xi_{*}(\widehat{\omega}) \cdot e_{1} .
\end{aligned}
$$


For the next theorem on the properties of $\xi_{*}$, recall $\mathscr{U}_{\xi_{*}}(\widehat{\omega})=\left[\xi_{*}(\widehat{\omega}), \bar{\xi}_{*}(\widehat{\omega})\right]$ from (2.7).

Theorem 5.3 Assume $\mathbb{P}\left\{\omega_{0} \leq r\right\}$ is continuous in $r$. Then on the extended space $(\widehat{\Omega}, \widehat{\mathfrak{S}}, \widehat{\mathbb{P}})$ of Theorem 3.3 the random variable $\xi_{*}(\widehat{\omega}) \in \mathrm{ri} \mathscr{U}$ defined by $(5.2)$ has the following properties.

(i) $\widehat{\mathbb{P}}$-almost surely, for every $z \in \mathbb{Z}^{2}$, there exists a $\mathscr{U}_{\xi_{*}}\left(T_{z} \widehat{\omega}\right)$--directed geodesic out of $z$ that goes through $z+e_{2}$ and a $\mathscr{U}_{\xi_{*}}\left(T_{z} \widehat{\omega}\right)+$-directed geodesic out of $z$ that goes through $z+e_{1}$. The two geodesics intersect only at their starting point $z$.

(ii) The following holds $\widehat{\mathbb{P}}$-almost surely. Let $x_{0, \infty}^{\prime}$ and $x_{0, \infty}^{\prime \prime}$ be any geodesics out of 0 with

$$
\underline{\lim }_{n \rightarrow \infty} \frac{x_{n}^{\prime} \cdot e_{1}}{n}<\underline{\xi}_{*}(\widehat{\omega}) \quad \text { and } \quad \varlimsup_{n \rightarrow \infty} \frac{x_{n}^{\prime \prime} \cdot e_{1}}{n}>\bar{\xi}_{*}(\widehat{\omega}) .
$$

Then $x_{1}^{\prime}=e_{2}$ and $x_{1}^{\prime \prime}=e_{1}$.

(iii) $\xi_{*}$ is almost surely an exposed point (see (2.3) for the definition). Furthermore, $\widehat{\mathbb{P}}\left\{\widehat{\omega}: \xi_{*}(\widehat{\omega})=\xi\right\}>0$ if and only if $\xi \in($ ri $\mathscr{U}) \backslash \mathscr{D}$.

(iv) Fix $u \in \mathbb{Z}^{2}$.

(a) Let $\zeta, \eta \in$ ri $\mathscr{U}$ be such that $\zeta \cdot e_{1}<\eta \cdot e_{1}$ and $\nabla g_{\mathrm{pp}}(\zeta+) \neq \nabla g_{\mathrm{pp}}(\eta-)$. Then for $\widehat{\mathbb{P}}$-almost every $\widehat{\omega}$ there exists $z \in u+\mathbb{Z}_{+}^{2}$ such that $\left.\xi_{*}\left(T_{z} \widehat{\omega}\right) \in\right] \zeta, \eta[$.

(b) Let $\xi \in(\mathrm{ri} \mathscr{U}) \backslash \mathscr{D}$. Then for $\widehat{\mathbb{P}}$-almost every $\widehat{\omega}$ there exists $z \in u+\mathbb{Z}_{+}^{2}$ such that $\xi_{*}\left(T_{z} \widehat{\omega}\right)=\xi$.

The point $z$ can be chosen so that, in both cases (a) and (b), there are two geodesics out of $u$ that split at this $z$ and after that never intersect, and of these two geodesics the one that goes through $z+e_{2}$ is $\mathscr{U}_{\xi_{*}}\left(T_{z} \widehat{\omega}\right)-$-directed, while the one that goes through $z+e_{1}$ is $\mathscr{U}_{\xi_{*}}\left(T_{z} \widehat{\omega}\right)+$-directed.

Proof Fix a (possibly $\widehat{\omega}$-dependent) $z \in \mathbb{Z}^{2}$. Define

$$
\begin{aligned}
& B_{+}^{*}(\widehat{\omega}, x, y)=\lim _{\eta \cdot e_{1} \searrow \xi_{*}\left(T_{z} \widehat{\omega}\right) \cdot e_{1}} B_{ \pm}^{\eta}(\widehat{\omega}, x, y) \\
& \text { and } \quad B_{-}^{*}(\widehat{\omega}, x, y)=\lim _{\zeta \cdot e_{1}} \lim _{*}\left(T_{z} \widehat{\omega}\right) \cdot e_{1} \\
& B_{ \pm}^{\zeta}(\widehat{\omega}, x, y) .
\end{aligned}
$$

We have to keep the $B_{ \pm}^{*}$ distinction because the almost sure continuity statement (3.7) does not apply to the random direction $\xi_{*}$. In any case, $B_{ \pm}^{*}$ are additive (Definition 3.1(c)) and recover weights $\omega_{x}=\min _{i=1,2} B_{ \pm}^{*}\left(\widehat{\omega}, x, x+e_{i}\right)$. From (5.2) and stationarity (Definition 3.1(b)) we have

$$
B_{+}^{*}\left(z, z+e_{1}\right) \leq B_{+}^{*}\left(z, z+e_{2}\right) \quad \text { and } \quad B_{-}^{*}\left(z, z+e_{1}\right) \geq B_{-}^{*}\left(z, z+e_{2}\right) .
$$

Fix any two tie-breaking rules $\mathfrak{t}^{+}$and $\mathfrak{t}^{-}$such that $\mathfrak{t}^{+}(z)=e_{1}$ and $\mathfrak{t}^{-}(z)=e_{2}$. By Lemma 4.1 and (5.5) there exists a geodesic from $z$ through $z+e_{1}$ (by following minimal $B_{+}^{*}$ gradients and using rule $\mathfrak{t}^{+}$) and another through $z+e_{2}$ (by following minimal $B_{-}^{*}$ gradients and using rule $\mathfrak{t}^{-}$). These two geodesics cannot coalesce because $\omega_{0}$ has a continuous distribution. 
Let $\zeta \cdot e_{1}<\xi_{*}\left(T_{z} \widehat{\omega}\right) \cdot e_{1}$. By the limits in (5.4) and monotonicity (3.5),

$$
\text { and } \quad \begin{aligned}
B_{+}^{\zeta}\left(\widehat{\omega}, x, x+e_{1}\right) & \geq B_{-}^{*}\left(\widehat{\omega}, x, x+e_{1}\right) \geq B_{-}^{\xi_{*}\left(T_{z} \widehat{\omega}\right)}\left(\widehat{\omega}, x, x+e_{1}\right) \\
B_{+}^{\zeta}\left(\widehat{\omega}, x, x+e_{2}\right) & \leq B_{-}^{*}\left(\widehat{\omega}, x, x+e_{2}\right) \leq B_{-}^{\xi_{*}\left(T_{z} \widehat{\omega}\right)}\left(\widehat{\omega}, x, x+e_{2}\right) .
\end{aligned}
$$

These inequalities imply that the geodesics that follow the minimal gradients of $B_{-}^{*}$ stay to the right of $x_{0, \infty}^{z, \zeta,+}$ and to the left of $x_{0, \infty}^{z, t^{-}, \xi_{*}\left(T_{z} \widehat{\omega}\right),-}$. By Theorem 4.3 these latter geodesics are $\mathscr{U}_{\zeta+}$ - and $\mathscr{U}_{\xi_{*}}\left(T_{z} \widehat{\omega}\right)-$-directed, respectively. Taking $\zeta \rightarrow \xi_{*}\left(T_{z} \widehat{\omega}\right)$ shows the $B_{-}^{*}$-geodesics are $\mathscr{U}_{\xi_{*}}\left(T_{z} \widehat{\omega}\right)$--directed. A similar argument gives that $B_{+}^{*}$-geodesics are $\mathscr{U}_{\xi_{*}}\left(T_{z} \widehat{\omega}\right)+$-directed. Part (i) is proved.

In part (ii) we prove the first claim, the other claim being similar. The assumption allows us to pick a rational $\eta \in \operatorname{ri} \mathscr{U} \operatorname{such}$ that $\underline{\lim } x_{n}^{\prime} \cdot e_{1} / n<\eta \cdot e_{1} \leq \eta \cdot e_{1}<\xi_{*} \cdot e_{1}$. Since $\omega_{0}$ has a continuous distribution and geodesic $x_{0, \infty}^{0, \eta,-}$ is $\overline{\mathscr{U}}_{\eta-}$-directed, geodesic $x_{0, \infty}^{\prime}$ has to stay always to the left of it. (5.2) implies $x_{1}^{0, \eta,-}=e_{2}$. Hence also $x_{1}=e_{2}$. The claim is proved.

For part (iii) fix first $\xi \in \mathscr{D}$, which implies $B_{ \pm}^{\xi}=B^{\xi}$. By Lemma 4.7, $B^{\xi}\left(0, e_{1}\right) \neq$ $B^{\xi}\left(0, e_{2}\right)$ almost surely. Let $\zeta \cdot e_{1} \searrow \xi \cdot e_{1}$ along rational points $\zeta \in \operatorname{ri} \mathscr{U}$. By (3.6), $B_{ \pm}^{\zeta}\left(0, e_{i}\right) \rightarrow B^{\xi}\left(0, e_{i}\right)$ a.s. Then on the event $B^{\xi}\left(0, e_{1}\right)>B^{\xi}\left(0, e_{2}\right)$ there almost surely exists a rational $\zeta$ such that $\zeta \cdot e_{1}>\xi \cdot e_{1}$ and $B_{ \pm}^{\zeta}\left(0, e_{1}\right)>B_{ \pm}^{\zeta}\left(0, e_{2}\right)$. By (5.2) this forces $\xi_{*} \cdot e_{1} \geq \zeta \cdot e_{1}>\xi \cdot e_{1}$. Similarly on the event $B^{\xi}\left(0, e_{1}\right)<B^{\xi}\left(0, e_{2}\right)$ we have almost surely $\xi_{*} \cdot e_{1}<\xi \cdot e_{1}$. The upshot is that $\mathbb{P}\left(\xi_{*}=\xi\right)=0$.

Now fix $\xi \in($ ri $\mathscr{U}) \backslash \mathscr{D}$. By Lemma 5.2 there exists a $z$ such that with positive probability geodesics $x_{0, \infty}^{0, \xi, \pm}$ separate at $z$. This separation implies that $B_{-}^{\xi}\left(z, z+e_{2}\right)<$ $B_{-}^{\xi}\left(z, z+e_{1}\right)$ and $B_{+}^{\xi}\left(z, z+e_{1}\right)<B_{+}^{\xi}\left(z, z+e_{2}\right)$, which says that $\xi_{*}\left(T_{z} \widehat{\omega}\right)=\xi$ and thus $\xi$ is an atom of $\xi_{*}$. We have proved the second statement in part (iii).

The non-exposed points of ri $\mathscr{U}$ consist of open linear segments of $g_{\mathrm{pp}}$ and the endpoints of these segments that lie in $\mathscr{D}$. Consider a segment $[\zeta, \eta] \subset$ ri $\mathscr{U}$ on which $g_{\mathrm{pp}}$ is linear. Theorem 3.3(iii) says $B_{+}^{\zeta}=B^{\xi}=B_{-}^{\eta}$ for all $\left.\xi \in\right] \zeta, \eta[$. Hence the inequalities in (5.2) go the same way throughout the segment and therefore $\left.\xi_{*} \in\right] \zeta, \eta[$ has zero probability. Points in $\mathscr{D}$ were taken care of above. Since there are at most countably many linear segments, the first claim in part (iii) follows.

Part (iv). Assume first $\zeta \cdot e_{1}<\eta \cdot e_{1} \cdot \mathscr{U}_{\zeta+} \neq \mathscr{U}_{\eta-}$ and directedness (Theorem 4.3) force the cocycle geodesics $x_{0, \infty}^{u, \eta,-}$ and $x_{0, \infty}^{u, \zeta,+}$ to eventually separate. This is clear if $\bar{\zeta} \neq \eta$ because then $\mathscr{U}_{\zeta+}$ and $\mathscr{U}_{\eta-}$ are disjoint. If, on the other hand, $\bar{\zeta}=\eta=\xi$, then $\nabla g_{\mathrm{pp}} \overline{(\xi-)}=\nabla g_{\mathrm{pp}}(\zeta+)$ and $\nabla g_{\mathrm{pp}}(\xi+)=\nabla g_{\mathrm{pp}}(\eta-)$. By Theorem 3.3(iii) we have $x_{0, \infty}^{u, \zeta,+}=x_{0, \infty}^{u, \xi,-}$ and $x_{u, \infty}^{0, \eta,-}=x_{0, \infty}^{u, \xi,+}$. The separation claim then follows from Lemma 5.2 .

Now that we know the two geodesics separate at some random point $z$ we have almost surely $B_{+}^{\zeta}\left(z, z+e_{2}\right)<B_{+}^{\zeta}\left(z, z+e_{1}\right)$. By continuity (3.6) there is almost surely a rational $\zeta^{\prime} \in \operatorname{ri} \mathscr{U}$ with $\zeta^{\prime} \cdot e_{1}>\zeta \cdot e_{1}$ such that $B_{+}^{\zeta^{\prime}}\left(z, z+e_{2}\right)<B_{+}^{\zeta^{\prime}}\left(z, z+e_{1}\right)$. Now we have $\zeta \cdot e_{1}<\zeta^{\prime} \cdot e_{1} \leq \xi_{*}\left(T_{z} \widehat{\omega}\right) \cdot e_{1}$. A similar argument shows $\eta \cdot e_{1}>\xi_{*}\left(T_{z} \widehat{\omega}\right) \cdot e_{1}$. Thus $\left.\xi_{*}\left(T_{z} \widehat{\omega}\right) \in\right] \zeta, \eta[$. 
Recall $B_{ \pm}^{*}$ and $\mathfrak{t}^{ \pm}$defined at and below (5.4) in terms of this $z=z(\widehat{\omega})$. Consider two geodesics that start at $u$, follow minimal $B_{+}^{*}$ and $B_{-}^{*}$ gradients, and use tie-breaking rules $\mathfrak{t}^{+}$and $\mathfrak{t}^{-}$, respectively. By monotonicity (3.5) the two geodesics have to stay sandwiched between $x_{0, \infty}^{u, \zeta,+}$ and $x_{0, \infty}^{u, \eta,-}$ and therefore must pass through $z$. Beyond $z$ these two geodesics are the ones discussed in the proof of part (i).

In case (b) with $\xi \in($ ri $\mathscr{U}) \backslash \mathscr{D}$, Lemma 5.2 gives the separation of $x_{0, \infty}^{u, \xi, \pm}$ at some random $z$. Then $\xi_{*}\left(T_{z} \widehat{\omega}\right)=\xi$ and the geodesics claimed in the theorem are directly given by $x_{0, \infty}^{u, \xi, \pm}$.

The next theorem identifies the asymptotic direction of the competition interface $\varphi=\left(\varphi_{k}\right)_{0 \leq k<\infty}$ defined in Sect. 2.6.

Theorem 5.4 Assume $\mathbb{P}\left\{\omega_{0} \leq r\right\}$ is continuous in $r$.

(i) All limit points of the asymptotic velocity of the competition interface are in $\mathscr{U}_{\xi_{*}(\widehat{\omega})}:$ for $\widehat{\mathbb{P}}$-almost every $\widehat{\omega}$

$$
\underline{\xi}_{*}(\widehat{\omega}) \cdot e_{1} \leq \underline{\lim }_{n \rightarrow \infty} n^{-1} \varphi_{n}(\omega) \cdot e_{1} \leq \varlimsup_{n \rightarrow \infty} n^{-1} \varphi_{n}(\omega) \cdot e_{1} \leq \bar{\xi}_{*}(\widehat{\omega}) \cdot e_{1}
$$

(ii) If $g_{\mathrm{pp}}$ is differentiable at the endpoints of its linear segments then $\xi_{*}$ is $\mathfrak{S}$ measurable and gives the asymptotic direction of the competition interface: $\widehat{\mathbb{P}}$-almost surely

$$
\lim _{n \rightarrow \infty} n^{-1} \varphi_{n}(\omega)=\xi_{*}(\widehat{\omega})
$$

Proof By (5.2), if $\zeta \cdot e_{1}<\xi_{*}(\widehat{\omega}) \cdot e_{1}<\eta \cdot e_{1}$, then $x_{1}^{0, \zeta, \pm}=e_{2}$ and $x_{1}^{0, \eta, \pm}=e_{1}$. Since the path $\varphi$ separates the geodesics that go through $e_{1}$ and $e_{2}$, it has to stay between $x_{0, \infty}^{0, \zeta,+}$ and $x_{0, \infty}^{0, \eta,-}$. By Theorem 4.3 these geodesics are $\mathscr{U}_{\zeta+}$ and $\mathscr{U}_{\eta-}$ directed, and we have

$$
\underline{\zeta} \cdot e_{1} \leq \varliminf_{n \rightarrow \infty} n^{-1} \varphi_{n} \cdot e_{1} \leq \varlimsup_{n \rightarrow \infty} n^{-1} \varphi_{n} \cdot e_{1} \leq \bar{\eta} \cdot e_{1}
$$

Claim (5.6) follows by taking $\zeta$ and $\eta$ to $\xi_{*}$.

If $g_{\mathrm{pp}}$ is differentiable at the endpoints of its linear segments, these endpoints are not exposed. Since $\xi_{*}$ is exposed by Theorem 5.3(iii), we have $\xi_{*}=\bar{\xi}_{*}=\xi_{*}$ and (5.7) follows from (5.6). Furthermore, cocycles are $\mathfrak{S}$-measurable and hence so is $\xi_{*}$.

Proof of Theorem 2.6 Limit (2.17) is in (5.7). The fact that the limit lies in ri $\mathscr{U}$ is in the construction in the paragraph that contains (5.2), and the properties of the limit are in Theorem 5.3 parts (iii) and (iv).

Proof of Theorem 2.8 Under the assumption of differentiability at endpoints of linear segments, either $\mathscr{U}_{\xi}$ equals $\{\xi\}$ or $\mathscr{U}_{\xi}$ has no exposed points. Hence, by Theorem 5.3(iii), almost surely $\mathscr{U}_{\xi_{*}}=\left\{\xi_{*}\right\}$ and $\xi_{*}\left(T_{x} \widehat{\omega}\right) \neq \xi$ implies that $\xi_{*}\left(T_{x} \widehat{\omega}\right) \notin \mathscr{U} \xi$. 
Consequently one of the cases in (5.3) covers simultaneously all $\mathscr{U} \xi$-directed geodesics in environment $T_{x} \widehat{\omega}$ and no separation at $x$ can happen for such geodesics. By Theorem 2.1(i) every geodesic is $\mathscr{U}_{\xi}$-directed for some $\xi \in \mathscr{U}$. One direction in part (i) is proved. The other direction comes from Theorem 5.3(i).

Part (ii) comes from part (i) and Theorem 5.3(iv).

As mentioned at the end of Sect. 2.6, if $\mathbb{P}\left\{\omega_{0} \leq r\right\}$ is not continuous in $r$, we have competition interfaces $\varphi^{(l)}$ and $\varphi^{(r)}$ for the trees of leftmost and rightmost geodesics. Their limiting directions $\xi_{*}^{(r)}(\widehat{\omega}), \xi_{*}^{(l)}(\widehat{\omega}) \in$ ri $\mathscr{U}$ are defined by

$$
\text { . } \begin{array}{ll}
B_{ \pm}^{\zeta}\left(\widehat{\omega}, 0, e_{1}\right)>B_{ \pm}^{\zeta}\left(\widehat{\omega}, 0, e_{2}\right) & \text { if } \zeta \cdot e_{1}<\xi_{*}^{(r)}(\widehat{\omega}) \cdot e_{1}, \\
& B_{ \pm}^{\zeta}\left(\widehat{\omega}, 0, e_{1}\right)=B_{ \pm}^{\zeta}\left(\widehat{\omega}, 0, e_{2}\right) \quad \text { if } \xi_{*}^{(r)}(\widehat{\omega}) \cdot e_{1}<\zeta \cdot e_{1}<\xi_{*}^{(l)}(\widehat{\omega}) \cdot e_{1} \\
\text { and } \quad B_{ \pm}^{\zeta}\left(\widehat{\omega}, 0, e_{1}\right)<B_{ \pm}^{\zeta}\left(\widehat{\omega}, 0, e_{2}\right) \text { if } \zeta \cdot e_{1}>\xi_{*}^{(l)}(\widehat{\omega}) \cdot e_{1} .
\end{array}
$$

With this definition limit (5.6) is valid also with superscripts $(l)$ and $(r)$. Consequently $n^{-1} \varphi_{n}^{(a)}(\omega) \rightarrow \xi_{*}^{(a)}(\widehat{\omega})$ a.s. for $a \in\{l, r\}$ under the assumption that $g_{\mathrm{pp}}$ is strictly concave.

\section{Exactly solvable models}

We derive here (2.18) for the distributions of $\xi_{*}^{(r)}$ and $\xi_{*}^{(l)}$ from definition (5.8). By Sections 3.1 and 7.1 of [23], $B^{(a, 1-a)}\left(0, e_{1}\right)$ and $B^{(a, 1-a)}\left(0, e_{2}\right)$ are independent geometric random variables with means

$$
\mathbb{E}\left[B^{\xi}\left(0, e_{j}\right)\right]=\mathbb{E}\left(\omega_{0}\right)+\sigma \sqrt{\xi \cdot e_{3-j} / \xi \cdot e_{j}}, \quad j=1,2
$$

The calculation for $\xi_{*}^{(r)}$ goes

$$
\begin{aligned}
\mathbb{P}\left\{\xi_{*}^{(r)} \cdot e_{1}>a\right\} & =\mathbb{P}\left\{B^{(a, 1-a)}\left(0, e_{1}\right)>B^{(a, 1-a)}\left(0, e_{2}\right)\right\} \\
& =\frac{\sqrt{\left(m_{0}-1\right)(1-a)}}{\sqrt{m_{0} a}+\sqrt{\left(m_{0}-1\right)(1-a)}}
\end{aligned}
$$

from which the first formula of (2.18) follows. Similar computation for $\xi_{*}^{(l)}$.

Acknowledgements N. Georgiou was partially supported by a Wylie postdoctoral fellowship at the University of Utah and the Strategic Development Fund (SDF) at the University of Sussex. F. Rassoul-Agha and N. Georgiou were partially supported by National Science Foundation Grant DMS-0747758. F. Rassoul-Agha was partially supported by National Science Foundation Grant DMS-1407574 and by Simons Foundation Grant 306576. T. Seppäläinen was partially supported by National Science Foundation Grants DMS1306777 and DMS-1602486, by Simons Foundation Grant 338287, and by the Wisconsin Alumni Research Foundation. 


\section{A Auxiliary technical results}

Cocycles satisfy a uniform ergodic theorem. The following is a special case of Theorem 9.3 of [24]. Note that a one-sided bound suffices for a hypothesis. Recall Definition 3.1 of stationary $L^{1}(\mathbb{P})$ cocycles. Let $h(B) \in \mathbb{R}^{2}$ denote the vector that satisfies

$$
\mathbb{E}\left[B\left(0, e_{i}\right)\right]=-h(B) \cdot e_{i} \quad \text { for } i \in\{1,2\} .
$$

Theorem A.1 Assume $\mathbb{P}$ is ergodic under the group $\left\{T_{x}\right\}_{x \in \mathbb{Z}^{2}}$. Let $B$ be a stationary $L^{1}(\mathbb{P})$ cocycle. Assume there exists a function $V$ such that for $\mathbb{P}$-a.e. $\omega$

$$
\varlimsup_{\varepsilon \searrow 0} \varlimsup_{n \rightarrow \infty} \max _{x:|x|_{1} \leq n} \frac{1}{n} \sum_{0 \leq k \leq \varepsilon n}\left|V\left(T_{x+k e_{i}} \omega\right)\right|=0 \quad \text { for } i \in\{1,2\}
$$

and $\max _{i \in\{1,2\}} B\left(\omega, 0, e_{i}\right) \leq V(\omega)$. Then

$$
\lim _{n \rightarrow \infty} \max _{\substack{x=z_{1}+\cdots+z_{n} \\ z_{1, n} \in\left\{e_{1}, e_{2}\right\}^{n}}} \frac{|B(\omega, 0, x)+h(B) \cdot x|}{n}=0 \quad \text { for } \mathbb{P} \text {-a.e. } \omega .
$$

If the process $\left\{V\left(T_{x} \omega\right): x \in \mathbb{Z}^{2}\right\}$ is i.i.d., then a sufficient condition for (A.1) is $\mathbb{E}\left(|V|^{p}\right)<\infty$ for some $p>2$ [40, Lemma A.4].

The following is a deterministic fact about gradients of passage times. This idea has been used profitably in planar percolation, and goes back at least to $[1,2]$. See Lemma 6.3 of [23] for a proof.

Lemma A.2 Fix $\omega \in \Omega$. Let $u, v \in \mathbb{Z}_{+}^{2}$ be such that $|u|_{1}=|v|_{1} \geq 1$ and $u \cdot e_{1} \leq v \cdot e_{1}$. Then

$$
\begin{aligned}
& G_{0, u}-G_{e_{1}, u} \geq G_{0, v}-G_{e_{1}, v} \text { and } \\
& G_{0, u}-G_{e_{2}, u} \leq G_{0, v}-G_{e_{2}, v} .
\end{aligned}
$$

\section{References}

1. Alm, S.E.: A note on a problem by Welsh in first-passage percolation. Combin. Probab. Comput. 7(1), $11-15(1998)$

2. Alm, S.E., Wierman, J.C.: Inequalities for means of restricted first-passage times in percolation theory. Combin. Probab. Comput. 8(4), 307-315 (1999). Random graphs and combinatorial structures (Oberwolfach, 1997)

3. Auffinger, A., Damron, M.: Differentiability at the edge of the percolation cone and related results in first-passage percolation. Probab. Theory Related Fields 156(1-2), 193-227 (2013)

4. Auffinger, A., Damron, M., Hanson, J.: Limiting geodesics for first-passage percolation on subsets of $\mathbb{Z}^{2}$. Ann. Appl. Probab. 25(1), 373-405 (2015)

5. Bakhtin, Y.: Burgers equation with random boundary conditions. Proc. Am. Math. Soc. 135(7), 22572262 (2007) (electronic)

6. Bakhtin, Y.: The Burgers equation with Poisson random forcing. Ann. Probab. 41(4), 2961-2989 (2013)

7. Bakhtin, Y.: Inviscid burgers equation with random kick forcing in noncompact setting. Electron. J. Probab. 21, 1-50, paper no. 37 (2016). doi:10.1214/16-EJP4413 
8. Bakhtin, Y., Cator, E., Khanin, K.: Space-time stationary solutions for the Burgers equation. J. Am. Math. Soc. 27(1), 193-238 (2014)

9. Bakhtin, Y., Khanin, K.: Localization and Perron-Frobenius theory for directed polymers. Mosc. Math. J. 10(4), 667-686, 838 (2010)

10. Cator, E., Pimentel, L.P.R.: A shape theorem and semi-infinite geodesics for the Hammersley model with random weights. ALEA Lat. Am. J Probab. Math. Stat. 8, 163-175 (2011)

11. Cator, E., Pimentel, L.P.R.: Busemann functions and equilibrium measures in last passage percolation models. Probab. Theory Related Fields 154(1-2), 89-125 (2012)

12. Cator, E., Pimentel, L.P.R.: Busemann functions and the speed of a second class particle in the rarefaction fan. Ann. Probab. 41(4), 2401-2425 (2013)

13. Cohn, H., Elkies, N., Propp, J.: Local statistics for random domino tilings of the Aztec diamond. Duke Math. J. 85(1), 117-166 (1996)

14. Coupier, D.: Multiple geodesics with the same direction. Electron. Commun. Probab. 16, 517-527 (2011)

15. Damron, M., Hanson, J.: Busemann functions and infinite geodesics in two-dimensional first-passage percolation. Commun. Math. Phys. 325(3), 917-963 (2014)

16. Durrett, R., Liggett, T.M.: The shape of the limit set in Richardson's growth model. Ann. Probab. 9(2), 186-193 (1981)

17. W, E., Khanin, K., Mazel, A., Sinai, Y.: Invariant measures for Burgers equation with stochastic forcing. Ann. Math. (2) 151(3), 877-960 (2000)

18. Ferrari, P.A., Kipnis, C.: Second class particles in the rarefaction fan. Ann. Inst. H. Poincaré Probab. Statist. 31(1), 143-154 (1995)

19. Ferrari, P.A., Martin, J.B., Pimentel, L.P.R.: A phase transition for competition interfaces. Ann. Appl. Probab. 19(1), 281-317 (2009)

20. Ferrari, P.A., Pimentel, L.P.R.: Competition interfaces and second class particles. Ann. Probab. 33(4), 1235-1254 (2005)

21. Garet, O., Marchand, R.: Coexistence in two-type first-passage percolation models. Ann. Appl. Probab. 15(1A), 298-330 (2005)

22. Georgiou, N., Rassoul-Agha, F., Seppäläinen, T.: Geodesics and the competition interface for the corner growth model (2015). arXiv:1510.00860v1 (preprint)

23. Georgiou, N., Rassoul-Agha, F., Seppäläinen, T.: Stationary cocycles and Busemann functions for the corner growth model. Probab. Theory Relat. Fields (2016). doi:10.1007/s00440-016-0729-x

24. Georgiou, N., Rassoul-Agha, F., Seppäläinen, T., Yılmaz, A.: Ratios of partition functions for the log-gamma polymer. Ann. Probab. 43(5), 2282-2331 (2015)

25. Hoang, V.H., Khanin, K.: Random Burgers equation and Lagrangian systems in non-compact domains. Nonlinearity 16(3), 819-842 (2003)

26. Hoffman, C.: Coexistence for Richardson type competing spatial growth models. Ann. Appl. Probab. 15(1B), 739-747 (2005)

27. Hoffman, C.: Geodesics in first passage percolation. Ann. Appl. Probab. 18(5), 1944-1969 (2008)

28. Howard, C.D., Newman, C.M.: Geodesics and spanning trees for Euclidean first-passage percolation. Ann. Probab. 29(2), 577-623 (2001)

29. Iturriaga, R., Khanin, K.: Burgers turbulence and random Lagrangian systems. Commun. Math. Phys. 232(3), 377-428 (2003)

30. Jockusch, W., Propp, J., Shor, P.: Random domino tilings and the arctic circle theorem (1998). arXiv:math/9801068

31. Licea, C., Newman, C.M.: Geodesics in two-dimensional first-passage percolation. Ann. Probab. 24(1), 399-410 (1996)

32. Mairesse, J., Prabhakar, B.: The existence of fixed points for the $\cdot / G I / 1$ queue. Ann. Probab. 31(4), 2216-2236 (2003)

33. Marchand, R.: Strict inequalities for the time constant in first passage percolation. Ann. Appl. Probab. 12(3), 1001-1038 (2002)

34. Martin, J.B.: Limiting shape for directed percolation models. Ann. Probab. 32(4), 2908-2937 (2004)

35. Mountford, T., Guiol, H.: The motion of a second class particle for the TASEP starting from a decreasing shock profile. Ann. Appl. Probab. 15(2), 1227-1259 (2005)

36. Newman, C.M.: A surface view of first-passage percolation. In: Proceedings of the International Congress of Mathematicians, vols. 1, 2 (Zürich, 1994), pp. 1017-1023. Birkhäuser, Basel (1995) 
37. Pimentel, L.P.R.: Multitype shape theorems for first passage percolation models. Adv. Appl. Probab. 39(1), 53-76 (2007)

38. Pimentel, L.P.R.: Duality between coalescence times and exit points in last-passage percolation models. Ann. Probab. (2015). arXiv:1307.7769 (to appear)

39. Prabhakar, B.: The attractiveness of the fixed points of a $/ / G I / 1$ queue. Ann. Probab. 31(4), 2237-2269 (2003)

40. Rassoul-Agha, F., Seppäläinen, T., Yılmaz, A.: Quenched free energy and large deviations for random walks in random potentials. Commun. Pure Appl. Math. 66(2), 202-244 (2013)

41. Rost, H.: Nonequilibrium behaviour of a many particle process: density profile and local equilibria. Z. Wahrsch. Verw. Gebiete 58(1), 41-53 (1981)

42. Seppäläinen, T.: Hydrodynamic scaling, convex duality and asymptotic shapes of growth models. Markov Process. Related Fields 4(1), 1-26 (1998)

43. Seppäläinen, T.: Existence of hydrodynamics for the totally asymmetric simple $K$-exclusion process. Ann. Probab. 27(1), 361-415 (1999)

44. Wehr, J.: On the number of infinite geodesics and ground states in disordered systems. J. Stat. Phys. 87(1-2), 439-447 (1997)

45. Wehr, J., Woo, J.: Absence of geodesics in first-passage percolation on a half-plane. Ann. Probab. 26(1), 358-367 (1998)

46. Wüthrich, M.V.: Asymptotic behaviour of semi-infinite geodesics for maximal increasing subsequences in the plane. In: In and out of equilibrium (Mambucaba, 2000), Progr. Probab., vol. 51, pp. 205-226. Birkhäuser Boston, Boston (2002) 